\title{
SMRT and Illumina RNA sequencing reveal novel insights into the heat stress response and crosstalk with leaf senescence in tall fescue
}

Yiguang Qian ${ }^{1 \dagger}$, Liwen Cao ${ }^{2 \dagger}$, Qiang Zhang ${ }^{2}$, Maurice Amee ${ }^{2}$, Ke Chen ${ }^{3 *}$ and Liang Chen ${ }^{2,4^{*}}$

\begin{abstract}
Background: As a cool-season grass species, tall fescue (Festuca arundinacea) is challenged by increasing temperatures. Heat acclimation or activation of leaf senescence, are two main strategies when tall fescue is exposed to heat stress (HS). However, lacking a genome sequence, the complexity of hexaploidy nature, and the short read of second-generation sequencing hinder a comprehensive understanding of the mechanism. This study aims to characterize the molecular mechanism of heat adaptation and heat-induced senescence at transcriptional and post-transcriptional levels.

Results: Transcriptome of heat-treated $(1 \mathrm{~h}$ and $72 \mathrm{~h}$ ) and senescent leaves of tall fescue were generated by combining single-molecular real-time and Illumina sequencing. In total, 4076; 6917, and 11,918 differentially expressed genes (DEGs) were induced by short- and long-term heat stress (HS), and senescence, respectively. Venn and bioinformatics analyses of DEGs showed that short-term HS strongly activated heat shock proteins (Hsps) and heat shock factors (Hsfs), as well as specifically activated FK506-binding proteins (FKBPS), calcium signaling genes, glutathione S-transferase genes, photosynthesis-related genes, and phytohormone signaling genes. By contrast, long-term HS shared most of DEGs with senescence, including the up-regulated chlorophyll catabolic genes, phytohormone synthesis/degradation genes, stressrelated genes, and NACs, and the down-regulated photosynthesis-related genes, FKBPs, and catalases. Subsequently, transient overexpression in tobacco showed that FaHsfA2a (up-regulated specifically by short-term HS) reduced cell membrane damages caused by HS, but FaNACO29 and FaNAM-B1 (up-regulated by long-term HS and senescence) increased the damages. Besides, alternative splicing was widely observed in $\mathrm{HS}$ and senescence responsive genes, including Hsps, Hsfs, and phytohormone signaling/synthesis genes.

(Continued on next page)
\end{abstract}

\footnotetext{
* Correspondence: kechen@mail.scuec.edu.cn; chenliang888@wbgcas.cn

†Yiguang Qian and Liwen Cao contributed equally to this work.

${ }^{3}$ College of Resources and Environmental Science, Key Laboratory of Catalysis

and Energy Materials Chemistry of Ministry of Education \& Hubei Key

Laboratory of Catalysis and Materials Science, South-Central University for

Nationalities, Wuhan, People's Republic of China

${ }^{2}$ CAS Key Laboratory of Plant Germplasm Enhancement and Specialty

Agriculture, Wuhan Botanical Garden, The Innovative Academy of Seed Design, Chinese Academy of Sciences, Wuhan, People's Republic of China

Full list of author information is available at the end of the article
}

C C The Author(s). 2020 Open Access This article is licensed under a Creative Commons Attribution 4.0 International License, which permits use, sharing, adaptation, distribution and reproduction in any medium or format, as long as you give appropriate credit to the original author(s) and the source, provide a link to the Creative Commons licence, and indicate if changes were made. The images or other third party material in this article are included in the article's Creative Commons licence, unless indicated otherwise in a credit line to the material. If material is not included in the article's Creative Commons licence and your intended use is not permitted by statutory regulation or exceeds the permitted use, you will need to obtain permission directly from the copyright holder. To view a copy of this licence, visit http://creativecommons.org/licenses/by/4.0/ The Creative Commons Public Domain Dedication waiver (http://creativecommons.org/publicdomain/zero/1.0/) applies to the data made available in this article, unless otherwise stated in a credit line to the data. 
(Continued from previous page)

Conclusions: The short-term HS can stimulate gene responses and improve thermotolerance, but long-term HS is a damage and may accelerate leaf senescence. These results contribute to our understanding of the molecular mechanism underlying heat adaptation and heat-induced senescence.

Keywords: Tall fescue, Short- and long-term heat stress, Senescence, SMRT and Illumina RNA-Seq, Responsive genes, Alternative splicing

\section{Background}

Tall fescue (Festuca arundinacea) is one of the most important and widely used cool-season turfgrass and forage species all over the world [1]. The optimum temperature for tall fescue growth ranges from 15 to $25^{\circ} \mathrm{C}$. The high temperature will affect its growth and development [2, $3]$. One of the major symptoms of heat damage is premature leaf senescence. Leaf senescence is a critical concern for turfgrass because it negatively influences not only the forage production, but also aesthetic turf quality. However, because of increasing global temperature, an extreme high temperature greater than $35^{\circ} \mathrm{C}$ during summer is more frequent and extensively distributed. Heat stress (HS) has been a major threat for tall fescue.

As sessile organisms, plants are compelled to develop diverse systems to prevent damages caused by high temperatures [4]. The induction of heat shock proteins (Hsps), including Hsp100s, Hsp90s, Hsp70s, Hsp60s, and small Hsps under the control of heat stress transcription factors (Hsfs) has been reported to play a pivotal role in the heat stress response (HSR) and in acquired thermotolerance in plants [5-7]. In Arabidopsis thaliana, the complex transcriptional networks of HS in HsfA1-dependent or independent pathways, have been elucidated [8]. Reactive oxygen species (ROS) scavenging enzymes and plant hormones were also shown to participate in heat stress response [9-11]. However, investigation on regulatory networks during HSR was mainly focused on model plants Arabidopsis and tomato. For tall fescue, most studies were about genome-wide or certain gene family expression profiles of HSR by transcriptional analysis. The expression levels of FaHsfs, especially FaHsfA2s, and the target genes of FaHsfs, including Hsps, ascorbate peroxidase (APX), inositol-3-phosphate synthase (IPS), and galactinol synthase (GOLS1) were significantly up-regulated by HS [12]. In addition, genes involved in cell division, cell cycle, cell maintenance, photosynthesis, protein synthesis, signaling, metabolism, hormone metabolism, and DNA, RNA and protein degradation were dramatically responsive to HS in tall fescue with opposite thermotolerance or with different heat treatments [13-15]. However, because of the complex genome background and the difficulty in developing the transgenic system, functional analysis of Hsfs, Hsps or heatrelated genes in tall fescue has been hardly reported [16, 17]. Only one FaHsfA2c was demonstrated to enhance heat tolerance of transgenic tall fescue through activating Hsps [16]. Although the research on HSR in tall fescue has advanced persistently in recent years, the similarity and difference between short- and long-term HSRs in tall fescue are still unclear.

As the major symptom of heat injury, heat-induced leaf senescence in cool-season grass species is of wide concern. Heat-induced leaf senescence was positively associated with ethylene and abscisic acid (ABA), and negatively associated with cytokinin synthesis in Bentgrass, and it can be suppressed through a transformation with cytokinin synthesis gene IPT $[18,19]$. The metabolic and protein profiling identified that the content of certain acids, sucrose, and monosaccharides, proteins in photosynthesis, and amino acid metabolism were related to the alleviation of heat-induced senescence by cytokinin and ethylene inhibitor [20, 21]. However, all these studies were performed in Bentgrass, and the relationship between heat stress response and leaf senescence at the transcriptional level in tall fescue is still unclear.

In addition to transcriptional changes, post-transcriptional changes generated by alternative splicing (AS) also play important roles in HSR and senescence [22-25]. HS-induced AS has been demonstrated as a common feature among $H s f s$ in Arabidopsis [26]. HS post-transcriptionally regulated the expression of HsfA2 by AS. However, there is still no study about the role of AS in heat stress response in tall fescue. Furthermore, the role of AS in senescence response has only been studied in animals and humans but not in plants. Therefore, the genome-wide response of AS to HS and HSinduced senescence in tall fescue is worth exploring.

Tall fescue is an allohexaploid ( $2 \mathrm{n}=42$, PPG1G1G2G2) with a genome size of about 5.50 million $\mathrm{Kb}$. The $\mathrm{P}$ genome comes from $F$. pratensis and the G1G2 genome is from $F$. arundinacea var. glaucescens auct [27]. The highly complex genome constitution, the absence of reference genome sequences, and the short-read length of Illumina sequencing have posed large challenges for exploring HS and senescence response in tall fescue by second-generation sequencing technology. Single-molecular real-time (SMRT) sequencing which generates full-length transcripts and constructs reference unigenes directly without further assembly can resolve these difficulties [28]. Also, SMRT sequencing technology can identify alternative isoforms produced by AS with higher accuracy [29]. In this study, we aimed to 
investigate the similarity and difference between short- and long-term HSR, and explore the relationship between HS and leaf senescence in tall fescue at the transcriptional and post-transcriptional level. Therefore, both Pacific Biosciences (PacBio) SMRT and Illumina-based sequencing of the transcriptome of 'Houndog 5' under control (Con), $1 \mathrm{~h}$ of heat treatment (HT_1h), $72 \mathrm{~h}$ of heat treatment (HT_ $72 \mathrm{~h}$ ), and natural senescence (Sen) conditions were performed. Our results help elucidate the underlying mechanism of HSR and heat-accelerated leaf senescence in tall fescue, and can provide important clues for in-depth characterization of heat-resistance breeding candidate genes in tall fescue.

\section{Results}

\section{Effects of heat stress and senescence on physiological} indexes

To determine the physiological changes caused by heat stress and senescence, electrolyte leakage (EL), and Fv/Fm were measured under control, short-term HS, long-term HS, and natural senescence conditions, respectively (Fig. 1). The EL is used to represent cell membrane damage. As expected, EL was increased with the increment of heat treatment time. The EL of senescent leaves was significantly higher than that in control green leaves as well. $\mathrm{Fv} / \mathrm{Fm}$ reflects the maximum quantum efficiency of photosystem II (PSII) photochemistry. After long-term heat treatment, Fv/Fm was significantly decreased. Likewise, Fv/Fm significantly declined in senescent leaves.

\section{A global description of full-length transcriptome}

To comprehensively investigate variation in expression of genes responsive to $\mathrm{HS}$ and senescence in tall fescue, we sequenced 12 mRNA samples from leaves of 'Houndog 5' at different developmental stages or under different times of HS treatments using Illumina HiSeq platform (secondgeneration sequencing). To obtain a more comprehensive reference transcriptome in absence of a reference genome of tall fescue, full-length mRNA sequencing of mixed tall fescue RNA derived from above 12 mRNA samples was conducted on the PacBio RSII platform (Fig. 2). A total of 318,932 polymerase reads were generated (PRJNA647166). After filtering adaptors and low-quality reads (less than $50 \mathrm{bp}), 11,961,314$ subreads with a mean length of $1355 \mathrm{bp}$ were obtained. Then, all the subreads were processed into 286,049 circular consensus sequences (CCS) reads to further improve the data quality. Depending on whether 5 ' primer, 3' primer, and poly (A) sequences were detected, 235,889 full-length non-chimeric reads (FLNCs) were extracted from the CCS reads. These FLNCs were subsequently corrected using Iterative Clustering for Error Correction (ICE), LoRDEC and CD-HIT to eliminate up to $99.99 \%$ of sequencing errors. Finally, 62,443 unigenes with an average length of $2595 \mathrm{bp}$, N90 of $1282 \mathrm{bp}$ were successfully identified, and 23,310 of the unigenes were longer than $3000 \mathrm{bp}$.

To analyze the function of the 62,443 unigenes of tall fescue, functional annotations of all full-length isoforms were investigated using seven databases, including nonredundant protein sequence database $(\mathrm{Nr})$, nucleotide sequence database $(\mathrm{Nt})$, Pfam, clusters of orthologous groups of proteins (KOG/COG), Swiss-prot, Kyoto encyclopedia of genes and genomes (KEGG), and gene ontology (GO). In total, there were 55,075 unigenes $(88.20 \%)$ successfully annotated in at least one database, with 19,677 unigenes (31.51\%) in all seven databases (Additional file 1).

For GO analysis (Additional file 2), genes involved in 'metabolic process' $(15,300)$, 'cellular process' $(14,564)$, and 'single-organism process' (8587) were highly represented in biological process. In terms of cellular component category, 'cell' (5842), 'cell part' (5842), and 'organelle' (3875) were highly enriched. Within the molecular function category, the major sub-categories were 'binding' $(19,433)$, 'catalytic activity' (14,076), and 'transporter activity' (1583).

For KOG analysis (Additional file 2), a total of 32,982 enriched unigenes were divided into 26 groups. The largest group was 'general function prediction only' (7157), followed by 'posttranslational modification, protein turnover, chaperones' (6310), and 'signal transduction mechanisms' (3536).

For KEGG analysis (Additional file 2), 50,614 unigenes were classified into six main biochemical pathways: 'cellular processes', 'environmental information processing, 'genetic information processing', 'metabolism', 'organismal systems', and 'human diseases'. Within the metabolism pathway, 'energy metabolism', 'carbohydrate metabolism', and 'global and overview maps' were prominently represented. The pathways related to 'organismal systems' included 'endocrine system', 'immune system', 'aging', and 'environmental adaptation'. All functional annotations provided important information for analyzing the processes and pathways involved in HSR and senescence.

\section{Alternative splicing analysis in heat stress response and senescence}

One of the most important features of PacBio sequencing is the ability to identify AS. Given that there was no reference genome information of tall fescue, we utilized the COGENT to partition non-redundant transcripts into putative gene families and reconstruct each family into one or several fulllength UniTransModels for further AS analysis. Of the 62, 443 transcripts, COGENT constructed 6297 gene families with two or more isoforms. After splicing junction analysis, 3537 genes were identified with AS, generating 8312 isoforms. Then, a total of 298 AS events, including skipping exon (SE), mutually exclusive exon (MX), alternative 5' splice-site (A5), alternative 3 ' splice-site (A3), retained intron (RI), alternative first exon (AF), and alternative last exon (AL) were detected with SUPPA. RI was the most 
(a)

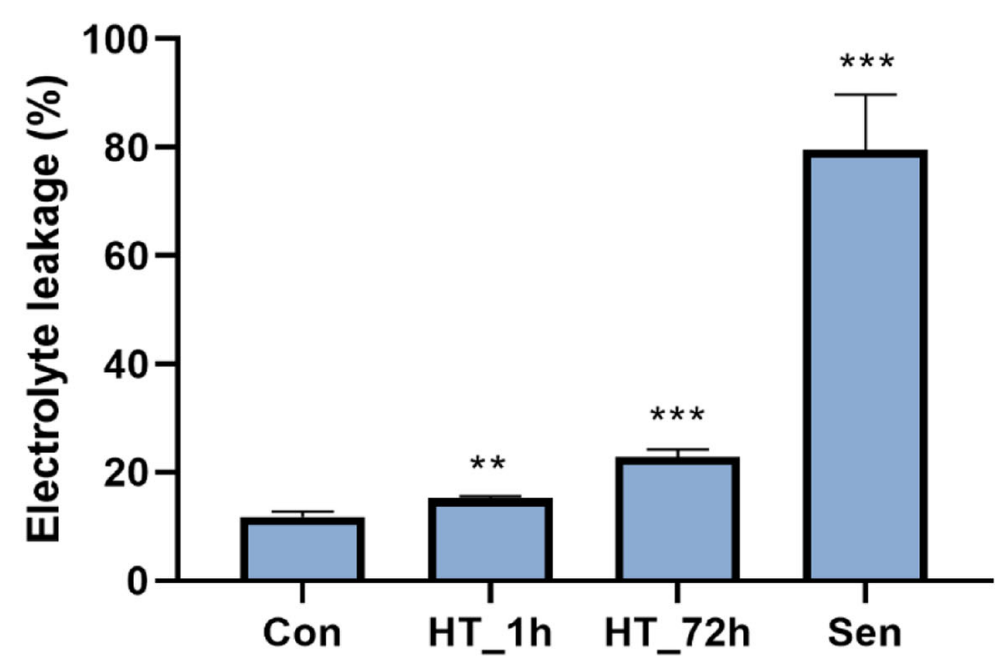

(b)

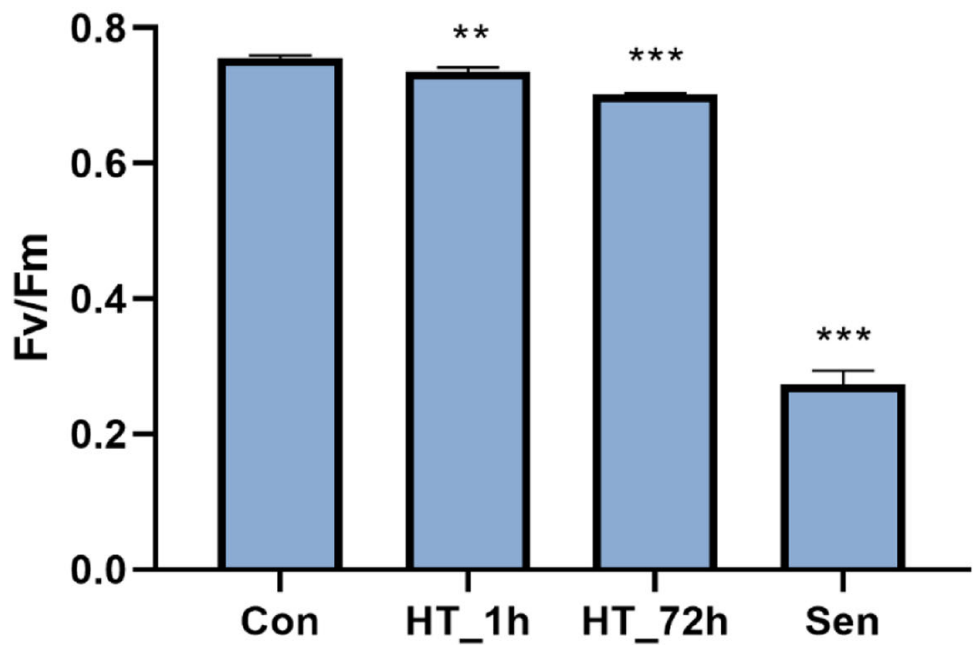

Fig. 1 Physiological variations induced by heat stress and senescence in tall fescue. a, b Electrolyte leakage (EL) (a) and PSII max-photo efficiencies (Fv/Fm) (b) of Con, HT_1h, HT_72h and Sen. Asterisks indicated significant difference with wild type at high temperature as estimated using Student's t-test $\left(^{* *} P<0.01,{ }^{* *} P<0.001\right)$. Error bars indicate the SD of three biological repeats. Con, control; $\mathrm{HT}_{-} 1 \mathrm{~h}, 1 \mathrm{~h}$ of $38^{\circ} \mathrm{C}$ heat treatment; HT_ $72 \mathrm{~h}, 72 \mathrm{~h}$ of $38^{\circ} \mathrm{C}$ heat treatment; Sen, senescent leaves

abundant AS events in tall fescue followed by A3 (Fig. 3a). Subsequently, the differential expression of AS events was analyzed. A total of 42,65 , and 43 differentially expressed AS events (DEASE) were identified in HT_1h vs Con, HT $72 \mathrm{~h}$ vs Con, and Sen vs Con, respectively (Fig. 3b).

\section{Analysis of differentially expressed genes and isoforms during $\mathrm{HS}$ and senescence}

To investigate the gene expression pattern under HS or natural senescence, DEGs were identified in three comparisons, including HT_1h vs Con, HT_72h vs Con, and Sen vs Con, with at least a two-fold difference and a $p$ - value less than 0.05 ( $\mid \log _{2}$ Ratio (Treatment/Control) $\mid \geq$ $1, p \leq 0.05)$. To reduce noise and improve the reliability of DEG analysis, the genes with below 10 FPKM in both control and treatment databases were all removed. As a result (Fig. 4a), a total of 4076 (2863 up-regulated genes and 1213 down-regulated genes), 6917 (2935 upregulated genes and 3982 down-regulated genes), and 11,918 (6760 up-regulated genes and 5158 downregulated genes) DEGs were detected in HT_1h vs Con, HT_72h vs Con, and Sen vs Con, respectively. Further analysis using a Venn diagram (Fig. 4b) showed that both unique and overlapping sets of DEGs were detected 


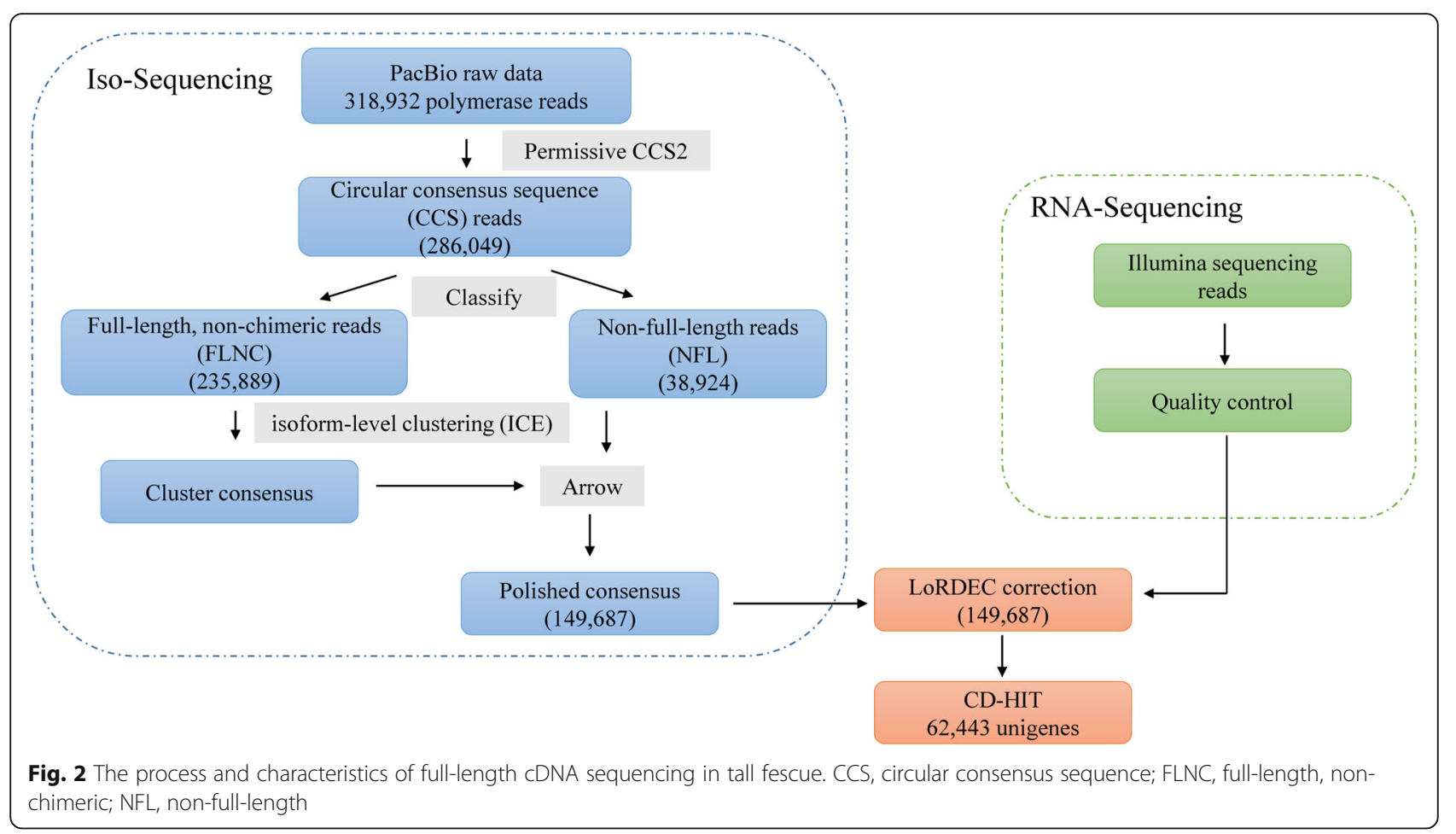

at each comparison. For the comparison of HT_72h vs Con, most DEGs (3835; 55.44\%) belonged to V5 (the intersection of HT_72h vs Con and Sen vs Con but HT $1 \mathrm{~h}$ vs Con). However, most DEGs $(2237 ; 54.88 \%)$ in the comparison of HT_1h vs Con were unique to HT_1h.

It has been known that post-transcriptional regulation, including alternative splicing can also change the expression of transcripts. Therefore, differentially expressed isoforms (DEIs, $\mid \log _{2}$ Ratio (Treatment/Control) $\mid \geq 1, p$-value $\leq 0.05$, FPKM $\geq 10$ ) produced by AS were also identified during heat stress and leaf senescence (Fig. 4c). Similar to DEGs, most DEIs $(293 / 529,55.39 \%)$ which were induced by HT $1 \mathrm{~h}$ were responsive to short-time heat stress specially, whereas most DEIs (465/900, 51.67\%) which were induced by HT_72h also responded to leaf senescence (Fig. $4 \mathrm{~d}$ ).

\section{Functional analysis of DEGs and DEls co-regulated by short- and long-term HS}

There were 413 DEGs in DEG_V4 (the intersection of comparison HT_1h vs Con and HT_72h vs Con but Sen vs Con), including 289 persistently up-regulated genes and 101 persistently down-regulated genes. Detailed metabolic pathway analysis (Fig. 5a) showed that most of these upregulated genes were significantly involved in protein processing in endoplasmic reticulum (125 DEGs), and spliceosome (17 DEGs). Further analysis found that the upregulated genes involved in the above pathways mainly encoded Hsps, including Hsp20, HspA5, Hsp90A, Hsp90B, and HspA1_8. They accounted for $43.60 \%$ (126/289) of the up-regulated DEGs. Heatmap (Fig. 5b, Additional file 3) of all induced Hsps showed that Hsps were strongly induced by HS, and the expressions of $H s p$ genes under short-term heat treatment were always higher than those under longterm heat treatment. Glutathione S-transferases (GSTs) are known to play a key role in the detoxification and reduction of ROS. A total of five genes encoding GSTs were upregulated by both short- and long-term HS (Fig. 5c, Additional file 3). In addition, eight genes involved in photosynthesis, including $p s a D, p s a L, p s b C, p s b R s, p s b W$, petE, and pet $F$ were responsive both to short- and long-term HS (Fig. $5 \mathrm{~d}$, Additional file 3). Among the 101 down-regulated genes, only one Hsp gene (i0_LQ_TF3rd_c75113/f1p27/ 835) was identified.

For DEI analysis, a total of 50 DEIs, including 28 persistently up-regulated isoforms and 17 persistently down-regulated isoforms were identified in DEI_V4 (the intersection of comparison HT_1h vs Con and HT_72h vs Con but Sen vs Con). Similar to DEGs in DEG_V4, half of up-regulated DEIs encoded Hsps.

\section{Short- and long-term HSR genes and isoforms encoded distinct functional groups}

Although there was an overlap, a set of genes exhibited altered expression patterns specific to short- or longterm HS. A total of 2237 genes and 293 isoforms were specifically responsive to HT_1h, most of which were upregulated $(2126 / 2237 ; 265 / 293)$. Among the upregulated genes and isoforms, 1626 DEGs and 193 DEIs exhibited at least 32-fold changes. The KEGG analysis showed that genes specifically induced by $\mathrm{HT}_{-} 1 \mathrm{~h}$ were 
(a)

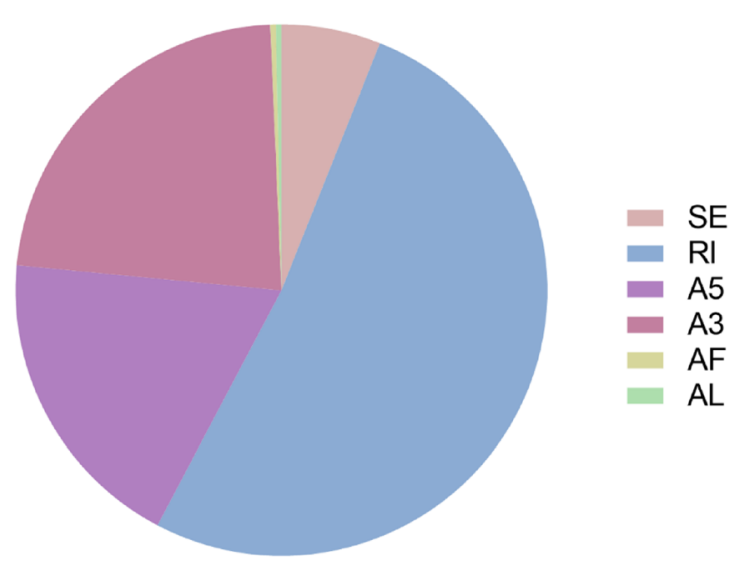

(b)

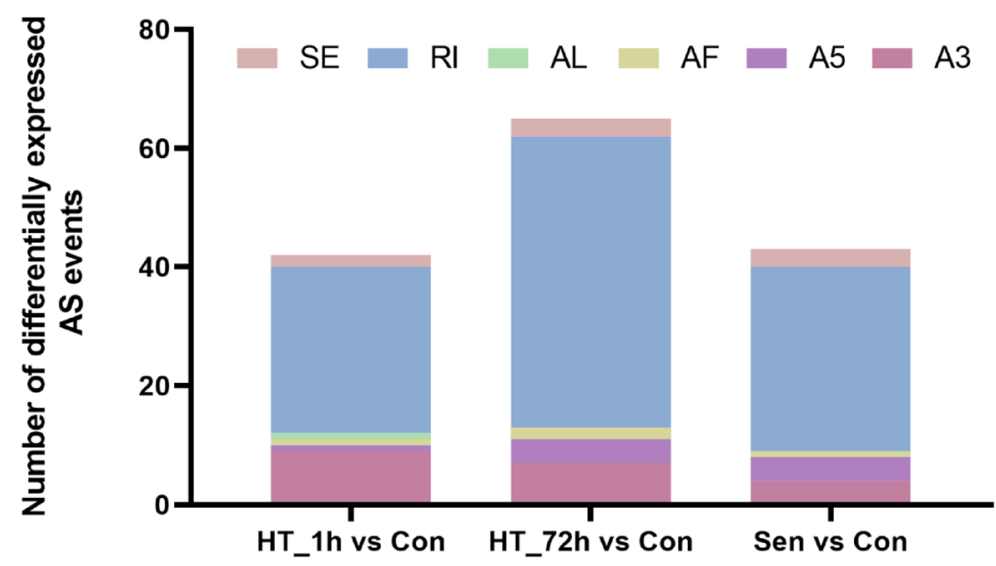

Fig. 3 Alternative splicing (AS) events analysis after heat stress treatments and natural senescence. a The number of six AS events in isoform sequencing (Iso-seq) sample. $\mathbf{b}$ The number of differentially expressed AS events in HT_1h vs Con, HT_72h vs Con, and Sen vs Con. SE, skipping exon; MX, mutually exclusive exon; A5, alternative 5' splice-site; A3, alternative 3' splice-site; Rl, retained intron; AF, alternative first exon; AL, alternative last exon

significantly involved in protein processing in endoplasmic reticulum, endocytosis, plant-pathogen metabolism, spliceosome, and protein export (Additional file 4a). DEGs enriched in these pathways almost all encoded Hsps. A total of 658 differentially expressed Hsp genes were identified, accounting for 30.95\% (Fig. 6a, Additional file 5). FK506-binding proteins (FKBPs), peptidylprolyl cis/trans isomerases, which have been reported to interact with HSP90 were also specifically induced by short-term heat stress (Fig. 6b, Additional file 5) [30]. After $1 \mathrm{~h}$ of heat treatment, six FKBP62, one FKBP65 and 42 FKBP70s were significantly up-regulated. In addition, several $\mathrm{Ca}^{2+}$ signaling genes were also significantly up-regulated after $1 \mathrm{~h}$ of heat treatment, including one Calcium-Dependent Protein Kinase 4 (CPK4), two Calcyclin Binding Proteins (Cacybps), one CalmodulinBinding Protein 60B (CBP60B), one Calmodulin-like Protein 15 (CML15), one CML16, one CML18, and one
CML25/26 (Fig. 6c, Additional file 5). Phytohormones play an important role in abiotic stress. Four genes participated in signal transduction of abscisic acid (ABA), jasmonic acid (JA) and cytokinin, including two PYL4s, one Coronatine Insensitive 1 (COI1), and one Histidine Phosphotransfer Protein $(A H P)$ were specifically responsive to short-term HS (Fig. 6d, Additional file 5). Then, the KEGG analysis of DEIs specifically induced by HT_1h was also performed. Protein processing in endoplasmic reticulum, plant-pathogen interaction, cutin, suberine and wax biosynthesis, and spliceosome were significantly enriched (Additional file 4b). The Hsps still accounted for the largest part of DEIs; however, the ratio $(20.38 \%, 54$ / 265) was much lower than that of DEGs.

After $72 \mathrm{~h}$ of high-temperature treatment, a total of 1336 DEGs and 197 DEIs were specially activated. Among the up-regulated DEGs and DEIs, only 104 DEGs and nine DEIs showed more than 32-fold changes, 


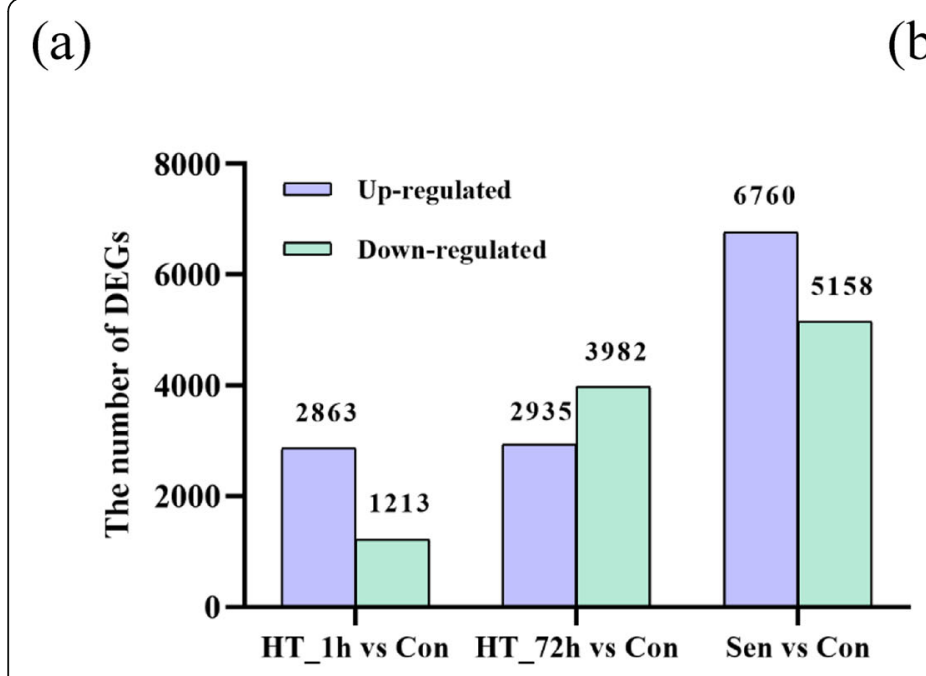

(b)
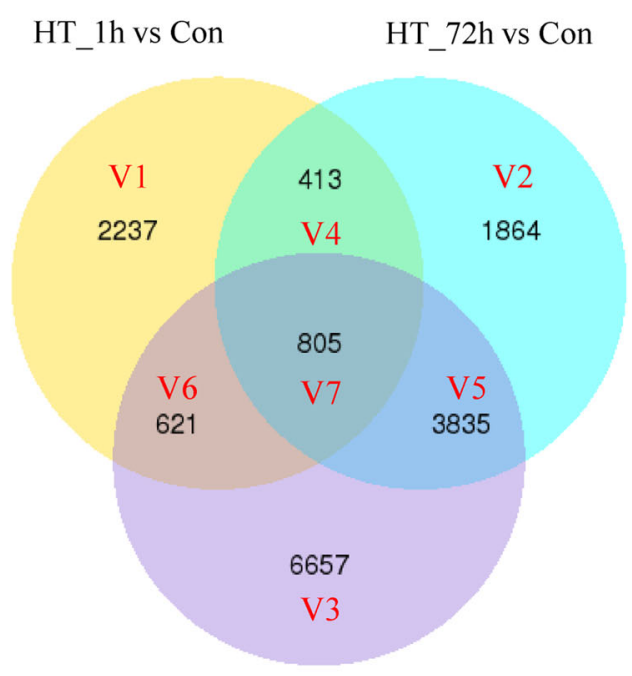

Sen vs Con

(c)

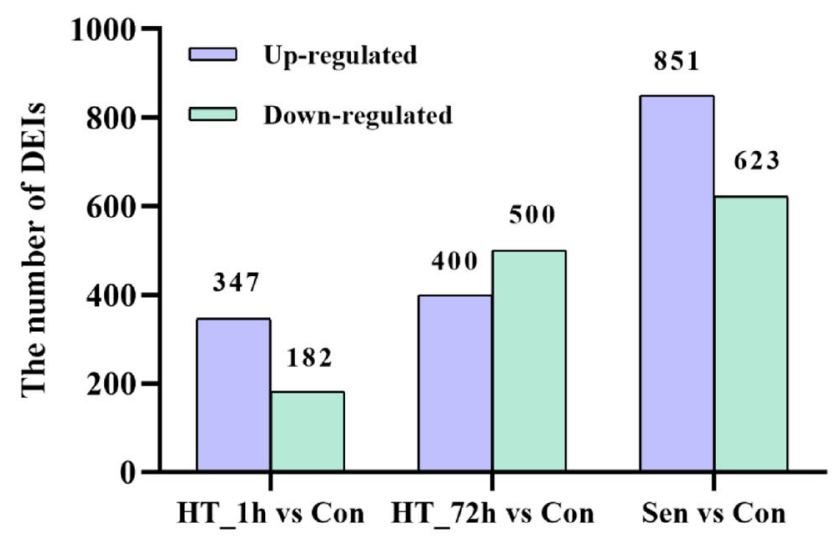

(d)
HT_1h vs Con HT_72h vs Con

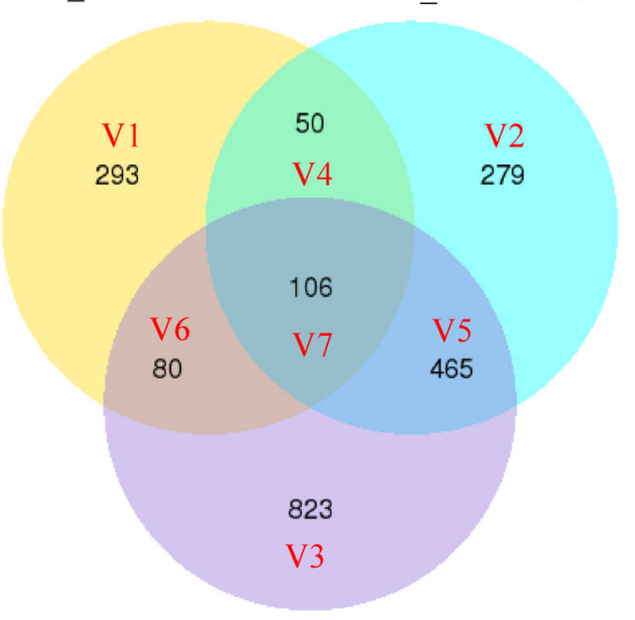

Sen vs Con

Fig. 4 Analysis of differentially expressed genes (DEGs) and isoforms (DEIs) in response to heat stress and natural senescence. a The number of up- and down-regulated DEGs at each time point of heat stress and senescence stage. $\mathbf{b}$ Venn diagram of DEGs in three comparisons. $\mathbf{c}$ The number of up- and down-regulated DEls at each time point of heat stress and senescence stage. $\mathbf{d}$ Venn diagram of DEls in three comparisons. V1, only HT_1h vs Con; V2, only HT_72h vs Con; V3, only Sen vs Con; V4, the intersection of HT_1h vs Con and HT_72h vs Con but Sen vs Con; V5, the intersection of HT_72h vs Con and Sen vs Con but HT_1h vs Con; V6, the intersection of HT_1h vs Con and Sen vs Con but HT_72h vs Con; V7, the intersection of HT_72h vs Con, Sen vs Con and HT_1h vs Con

the number of which was much less than that of DEGs specifically induced by HT_1h. Compared with genes induced by HT_1h, the molecular function of DEGs activated by HT_ $72 \mathrm{~h}$ was dramatically different. The most enriched pathways were ribosome, ribosome biogenesis in eukaryotes, RNA polymerase, pyrimidine metabolism, and purine metabolism (Additional file 6a). Only seven Hsps were specifically induced by HT_72h. A large number of ribosomal protein genes were specifically induced by HT_72h, including 23 small subunit ribosomal proteins (PRSs) and 39 large subunit ribosomal proteins (PRLs) (Fig. 7a, Additional file 7).
CYP genes, also encoding peptidyl-prolyl cis-trans isomerases, were specifically induced by $72 \mathrm{~h}$ of heat treatment (Fig. 7b, Additional file 7). In addition, six KT/HAK/KUP transporters, including one HAK10, two HAK18s, two HAK23s, and one HAK25 were identified in the DEG_V2 (Fig. 7c, Additional file 7). For DEIs specifically induced by HT_72h, no Hsps were identified. Differentially spliced CYPS, HAKs, and PRLS were significantly induced by $\mathrm{HT}_{-}$ $72 \mathrm{~h}$ as well. Unlike $1 \mathrm{~h}$ of heat treatment, relative more DEGs and DEIs were suppressed during $72 \mathrm{~h}$ of heat stress. A total of 735 DEGs and 100 DEIs were uniquely down- 


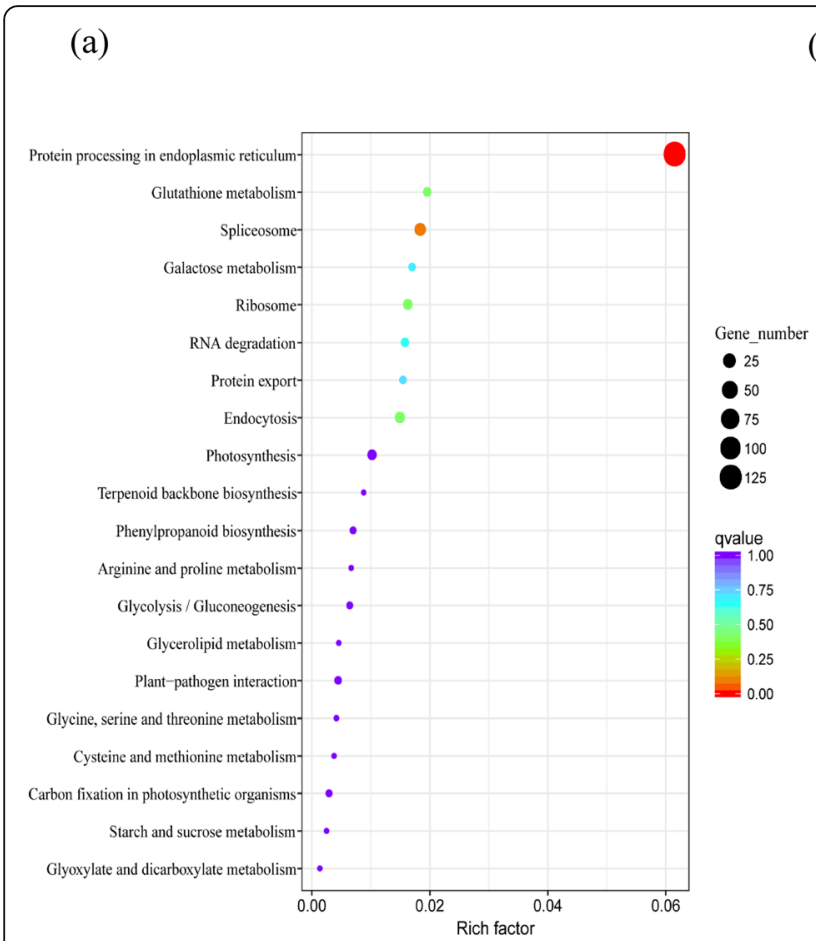

(b)

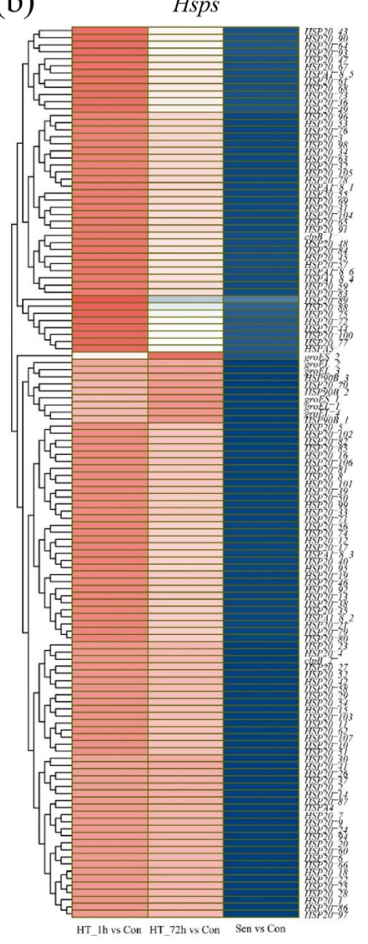

(c)

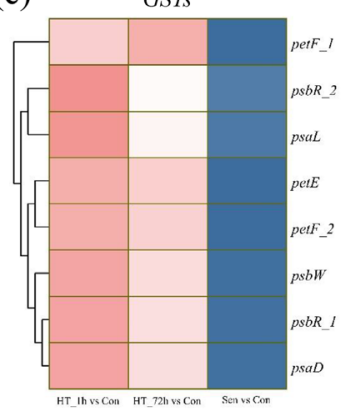

(d) DEGs involved in photosynthesis

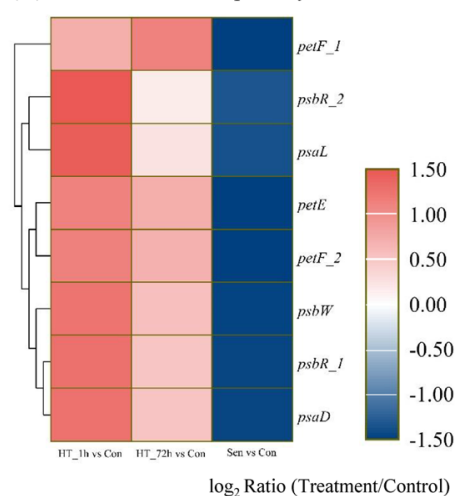

Fig. 5 Analysis of DEGs co-induced by HT_ih and HT_72h. a The top 20 KEGG pathways of up-regulated genes co-induced by short- and longterm heat treatments. b-d Hierarchical clustering analysis of Hsps (b), GSTs (c), and DEGs involved in photosynthesis (d) co-induced by short- and long-term heat stress during heat stress and senescence. The difference in expression is represented by a color block, and the color of the block ranges from blue (down-regulated) to red (up-regulated). Hsp, heat shock protein; GST, Glutathione S-transferase

regulated by HT_72h. The most enriched pathway was plant hormone signal transduction (Additional file 6b). A total of 22 DEGs involved in auxin, cytokinin, ABA, ethylene, and JA signal transduction were identified, such as Jasmonate ZIM-Domain (JAZ), Ethylene Insensitive 2 (EIN2), Small Auxin Up RNA 32 (SAUR32), etc (Fig. 7d, Additional file 7). Although several $\mathrm{Ca}^{2+}$ signaling genes were induced by $\mathrm{HT} \_1 \mathrm{~h}, \mathrm{Ca}^{2+}$ related genes were mainly down-regulated after $72 \mathrm{~h}$ of heat treatment (Fig. 7e, Additional file 7). In addition, sugar transporters, including three SWEET13s and one SWEET4, were specially suppressed by HT_72h (Fig. 7f, Additional file 7). Interestingly, seven 14-3-3 proteins (one GF14A, four GF14Bs, and two GF14Es) were identified as DEGs uniquely down-regulated after $72 \mathrm{~h}$ of heat treatment (Fig. 7g, Additional file 7). In terms of DEIs specifically suppressed by HT_72h, several DEIs involved in plant hormone signal transduction and $\mathrm{Ca}^{2+}$ signaling were generated by alternative splicing.

\section{Analysis of DEGs and DEls co-regulated by senescence and $\mathrm{HS}$}

As previously reported, the sustained high temperatures could accelerate leaf senescence [18]. Here, we found that most of the genes $(55.44 \%, 3835 / 6917)$ and isoforms
(51.67\%, 465/900) responsive to HT_72h also responded to Sen (Fig. 4b). A total of 1193 genes and 164 isoforms were induced commonly by HT_72h and Sen. The marker gene of leaf senescence SAG12 was significantly induced by $72 \mathrm{~h}$ of heat stress and natural senescence. In addition, this up-regulated DEGs list contained four kinds of chlorophyll catabolic-related genes (Fig. 8a, Additional file 8), including seven staygreens (SGRs), one pheophorbide a oxygenase (PAO), one pheophytinase (PPH), and one chlorophyll $b$ reductase (NOL). Among these upregulated DEGs, five ethylene and JA biosynthesis genes, and one cytokinin oxidase gene were identified (Fig. 8b, Additional file 8), including one 1-aminocyclopropane-1carboxylate oxidase (ACO), two 12-oxophytodienoate reductase $1 \mathrm{~s}$ (OPR1s), one peroxisome defective 1 (PED1), and one cytokinin oxidase/dehydrogenase 4 (CKX4). In addition, stress-related genes were also identified, including three stress enhanced protein $2 s$ (SEP2s), two stress-related proteins (SRPS), and four universal stress proteins PHOS34s (Fig. 8c, Additional file 8). The annotation analysis of DEIs showed that one PAO, two SGRs and one PED1 were generated by AS.

The number of down-regulated genes (2546) and isoforms (299) were much more than those of up-regulated 


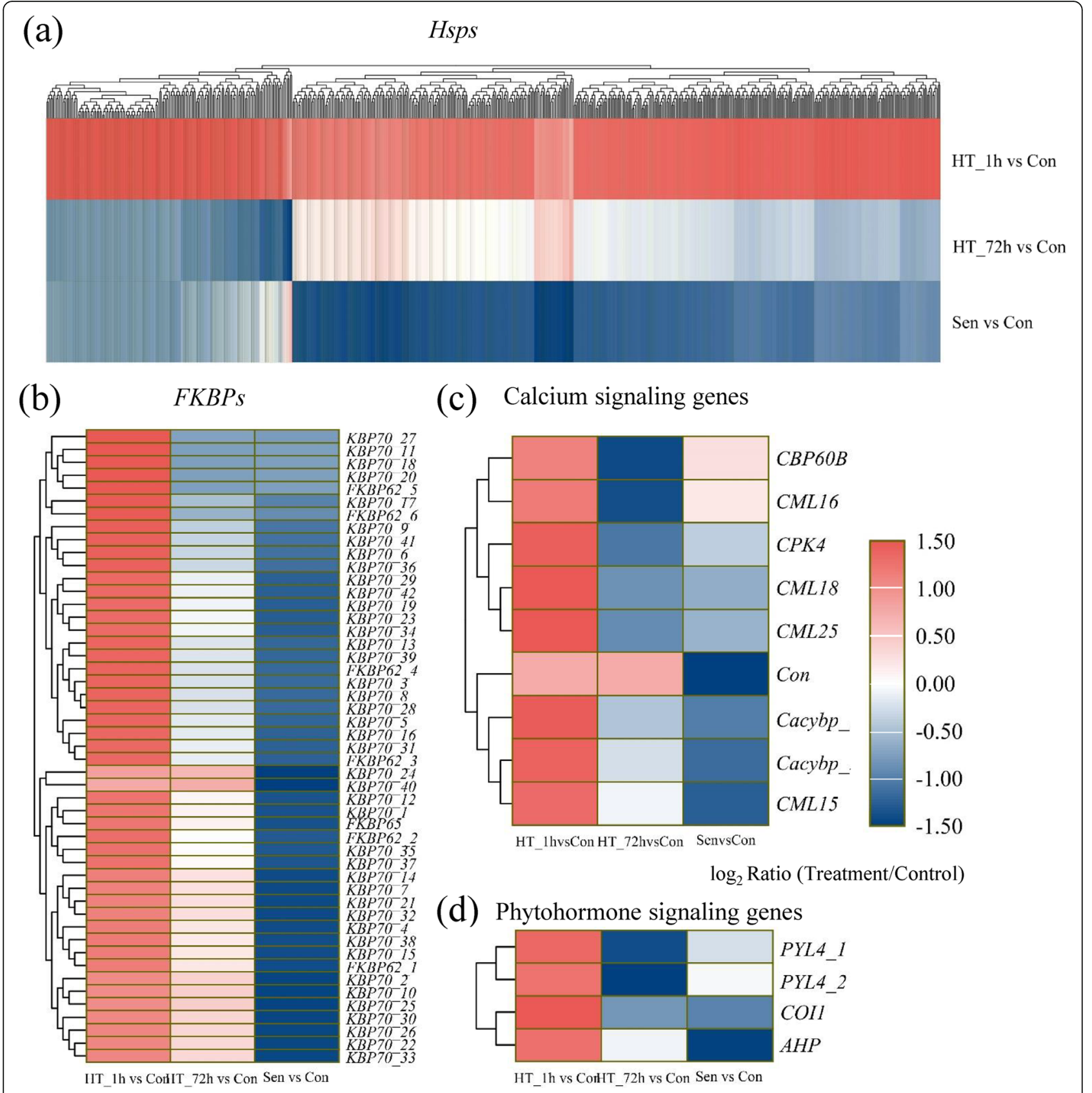

Fig. 6 Heatmaps of DEGs specifically induced by HT_1h. a-d Hierarchical clustering analysis of Hsps (a), FKBPs (b), calcium signaling genes (c), and phytohormone signaling genes (d) specifically up-regulated by HT_1h during heat stress and senescence. The difference in expression is represented by a color block, and the color of the block ranges from blue (down-regulated) to red (up-regulated). FKBP, FK506-binding proteins

genes and isoforms in V5. KEGG analysis showed that the most three enriched pathways were photosynthesisantenna proteins (176 DEGs), photosynthesis (205 DEGs), and carbon fixation in photosynthetic organisms (147 DEGs) (Additional file 9). DEGs involved in these pathways included a large number of $L H C A, L H C B$, $p s b A, p s b P, p s b W, p s b R, r b c S$, etc. Interestingly, FKBPs which were specifically induced by HT_1h were suppressed by HT_72h and Sen (Fig. 8d, Additional file 8).
In addition, two Catalase $1 \mathrm{~s}(C A T 1 s)$ and one CAT2 were down-regulated (Fig. 8e, Additional file 8). The DEI annotation results showed that 18,11 , and 8 DEIs involved in carbon fixation in photosynthetic organisms, photosynthesis-antenna proteins, and photosynthesis were generated by AS, respectively.

It is worth noting that among the 621 genes in V6 (the intersection of $\mathrm{HT}_{-} 1 \mathrm{~h}$ vs Con and Sen vs Con but HT_ $72 \mathrm{~h}$ vs Con), 93 DEGs showed the opposite variation 


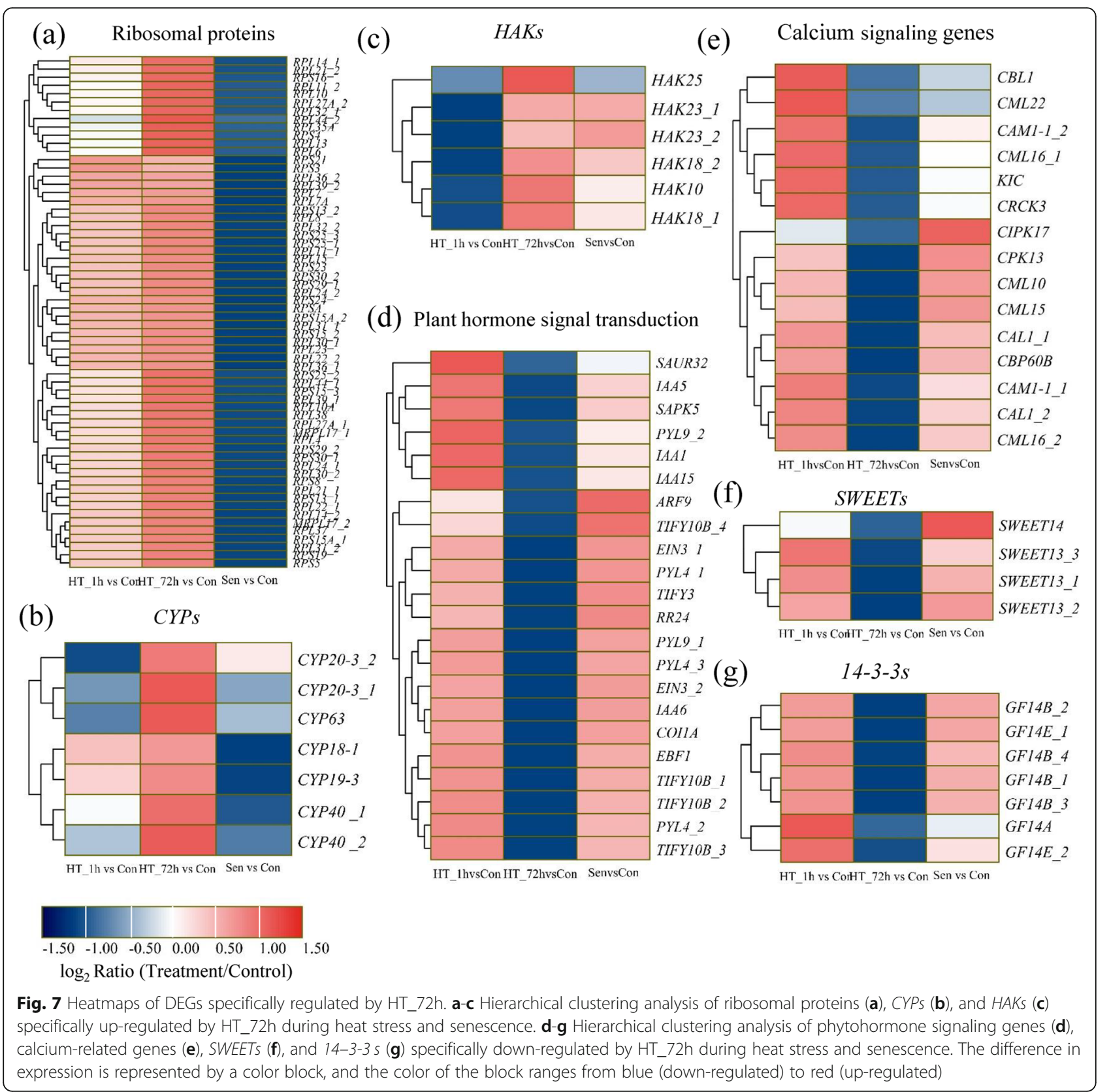

tendency between HT_1h vs Con and Sen vs Con, indicating that there was an antagonistic relationship between senescence and short-term HS. A total of 26 genes were significantly induced by HT_lh, but were suppressed by Sen. On the contrary, a total of 67 genes were significantly repressed by HT_1h, but were induced by Sen. Functional annotations showed that the heatinduced 26 DEGs contained three Hsps, three psbRs, one psbP, one GST, and one Uxin/Indole-3-Acetic Acid 3 (IAA3). The 67 senescence-induced DEGs contained two NRT1/PTR Family 6.4s (NPF6.4s), two NDR1/HIN1-like 10s (NHL1Os), and one STP1. Interestingly, SAG12, the most widely used senescence-associated reference gene for characterizing leaf senescence, was observed in the repressed genes by HT_1h.

\section{Functional analysis of DEGs and DEls specifically responsive to natural senescence}

Besides, genes and isoforms specifically responsive to natural senescence were also investigated. A total of 5119 and 633 up-regulated DEGs and DEIs were identified, respectively. KEGG analysis showed that these up-regulated DEGs were enriched in 108 pathways. The most significantly enriched pathways were valine, leucine and isoleucine degradation, fatty acid degradation, and galactose metabolism (Additional file 10a). Among 108 pathways, 92 were the 


\section{(a) Chlorophyll catabolic-related genes}

\section{(d)}

FKBPS

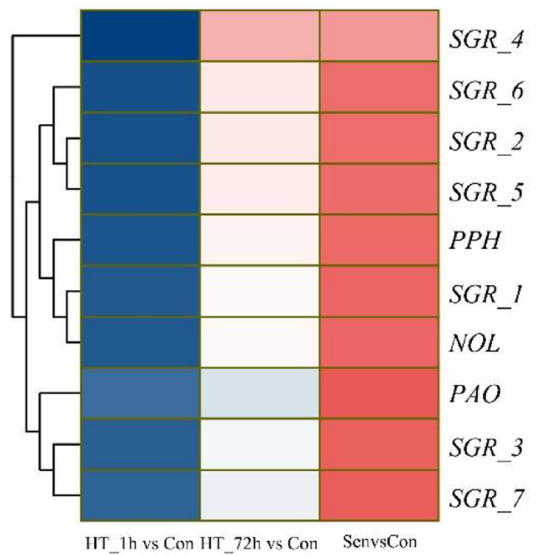

(b) Phytohormone synthesis

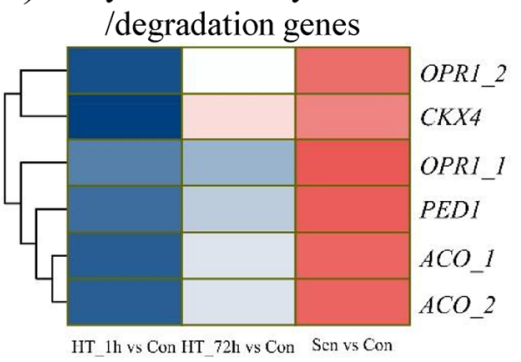

(c) Stress-related genes
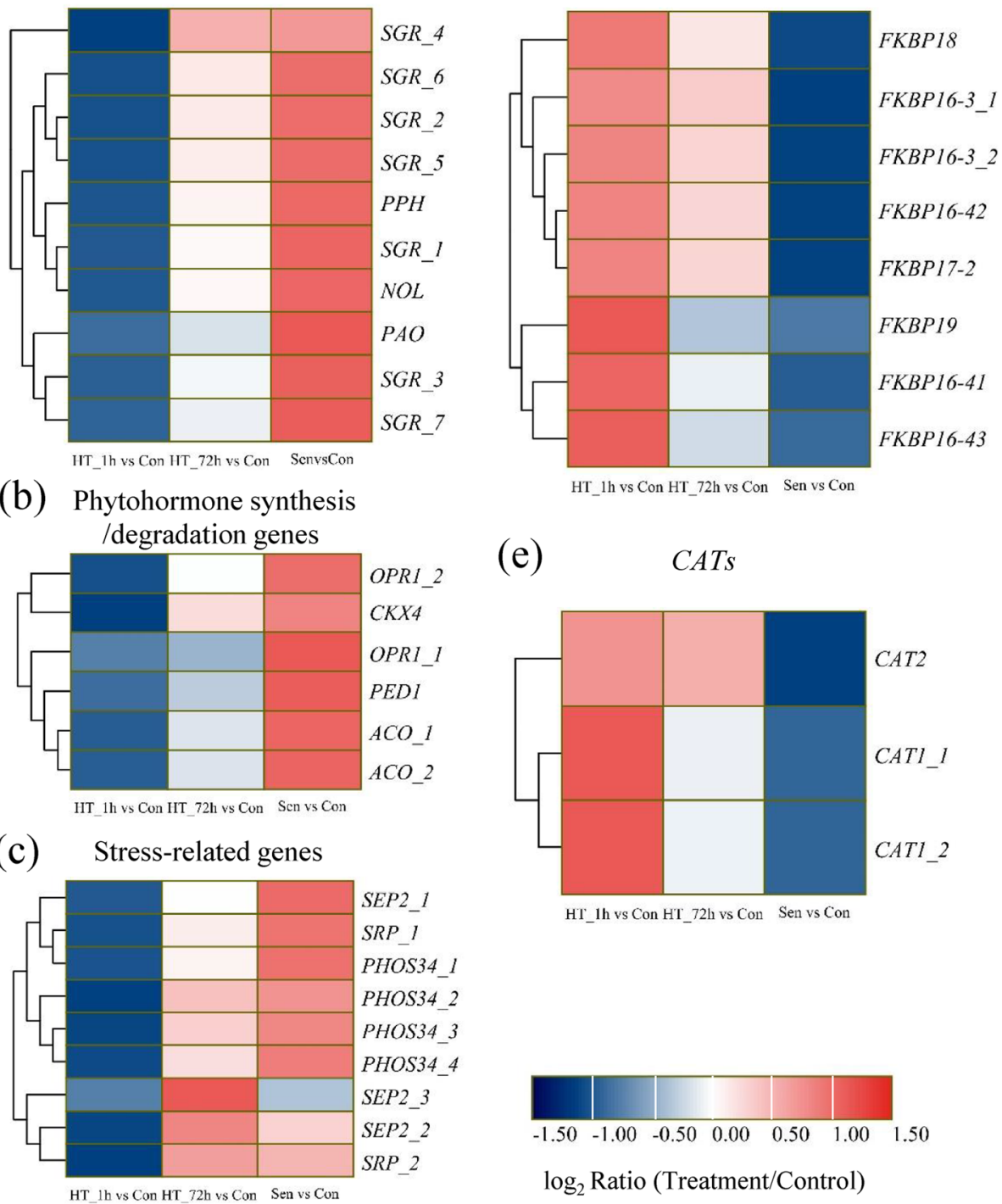

(e) $\quad C A T s$

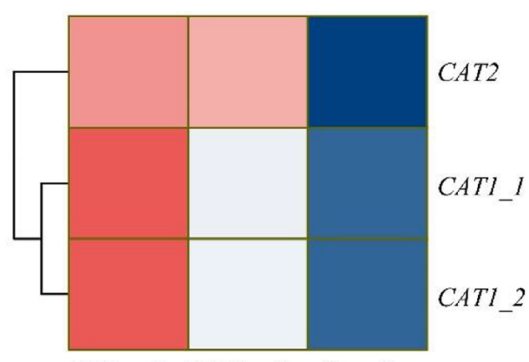

HT_th vs Con HT $72 \mathrm{~h}$ vs Con Sen vs Con

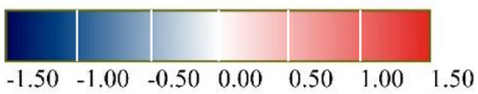

$\log _{2}$ Ratio (Treatment/Control)

Fig. 8 Heatmaps of DEGs co-regulated by HT_72h and Sen. a-c Hierarchical clustering analysis of chlorophyll catabolic-related genes (a), phytohormone synthesis/degradation genes $(\mathbf{b})$, and stress-related genes (c) co-induced by HT_72h and Sen during heat stress and senescence. d-g Hierarchical clustering analysis of FKBPS (d), and CATs (e) co-suppressed by HT_72h and Sen during heat stress and senescence. The difference in expression is represented by a color block, and the color of the block ranges from blue (down-regulated) to red (up-regulated). CAT, catalase

same as those responsive both to HT_72h and Sen. For DEIs, some senescence-associated genes, including three early flowering $3 s$ (ELF3s), one $P A O$, and one abscisic aldehyde oxidase 3 ( $A A O 3$ ) were generated by AS.

However, unlike with DEGs responsive both to $\mathrm{HT}_{-}$ $72 \mathrm{~h}$ and Sen, much less down-regulated than upregulated DEGs were identified specifically by Sen. Although many down-regulated DEGs were still enriched in photosynthesis, carbon fixation in photosynthetic organisms, and glyoxylate and dicarboxylate metabolism, most down-regulated DEGs were enriched in the ribosome (Additional file 10b).

\section{Major transcription factors (TFs) in response to HS and senescence}

During HS and senescence, a series of TF families were identified. The major TF families presented were Hsf, and NAC families (Additional file 11, Additional file 12). $H s f s$ were highly responsive to short-term HS. A total of $70 \mathrm{Hsfs}$ were specifically induced by HT_1h. In addition, one $H s f$ was specifically up-regulated by $\mathrm{HT}_{-} 72 \mathrm{~h}$, four Hsfs were up-regulated by both HT_1h and HT_72h, and two Hsfs were up-regulated by both HT_72 and Sen. For the four Hsfs induced both by HT_1h and HT_72h, the expression abundance of HT_1h was much higher 
than that of HT_72h. Most of the induced Hsfs belonged to class A, including HsfA2s and HsfA6s. However, only one class $\mathrm{C} H s f$ was identified, and it was suppressed specifically by senescence. As shown in Additional file 12, $N A C s$ were mainly induced by senescence. Totally, 38 $N A C s$ were specifically induced by Sen, and 16 NACs were co-induced by HT_72h and Sen. Only one NAC gene was up-regulated by HT_lh specifically. In contrast, down-regulated $N A C$ genes were mainly identified under heat stress.

The subsequent AS analysis showed that $H s f s$ were only alternatively spliced during short-term heat stress, including six $H s f A 2 a s$, one $H s f A 2 d$, one $H s f A 2 e$, two HsfB2as, two $H s f B 2 b s$, one $H s f B 2 c$, and eight HsfA6as. However, NAC genes were alternatively spliced not only during senescence but also during long-term heat stress. For example, isoforms i2_LQ_TF3rd_c10489/f1p2/2143 (NAC071) and i2
LQ_TF3rd_c8329/f1p0/2237 (NAC074) were generated by alternative splicing in Sen. Isoforms i1_LQ_TF3rd_c49009/ f1p0/1737 (NAC068) and i1_HQ_TF3rd_c9464/f2p0/1360 (NAC079) were generated by alternative splicing in Sen and HT_72h.

To validate the results of the RNA sequencing, six DEGs, including $3 \mathrm{NACs}$ and $3 \mathrm{Hsfs}$, were selected for quantitative real-time PCR (qRT-PCR) (Fig. 9a). DEGs $i 1_{-}$ LQ_TF3rd_c8869/f1p0/1379 (NAM-B2), i1_HQ_TF3rd_ c26766/f2p2/1431 (NAC029), and i1_HQ_TF3rd_c44692/ f2p0/1636 (NAM-B1) were induced both by Sen and HT 72h. DEGs i1_LQ_TF3rd_c31016/f1p0/1406 (HsfA2d-1) and i1_LQ TF3rd c80591/f1p0/1429 (HsfA2d-2) were induced both by HT_1h and HT_72h. DEG i3_HQ_TF3rd_ $c 12860 / f 2 p 0 / 3466$ annotated as $H s f A 2 a$ was specifically induced by HT_1h. As shown in Fig. 9a, the qRT-PCR results of all six DEGs were consistent with those obtained (a)

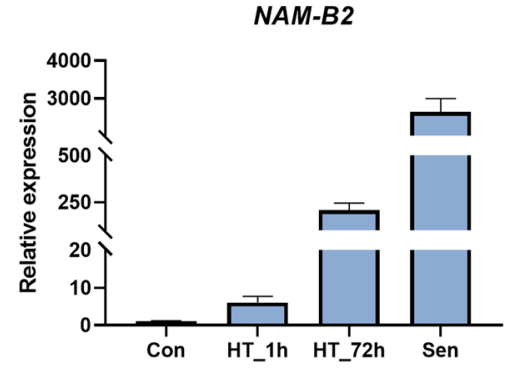

HsfA2d-1

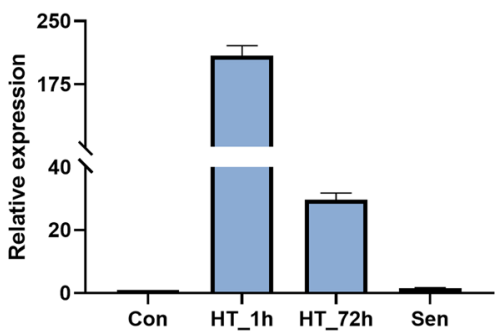

(b)

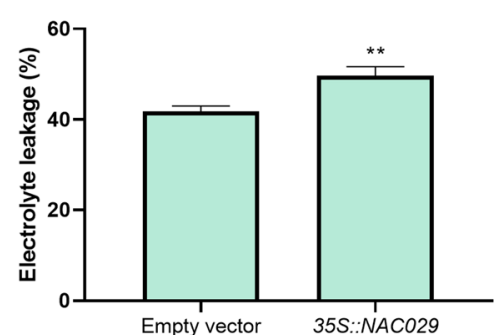

NAC029

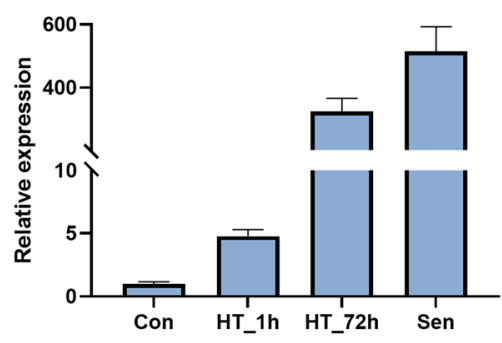

HsfA2d-2

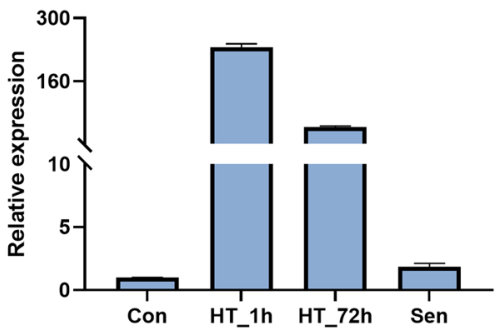

NAM-B1

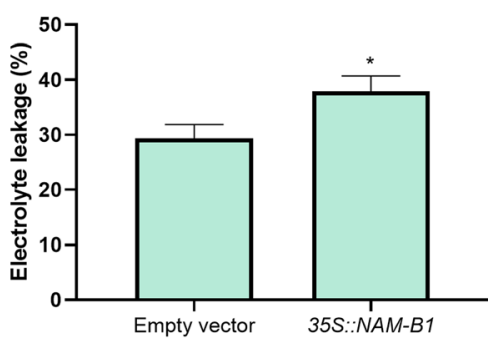

NAM-B1
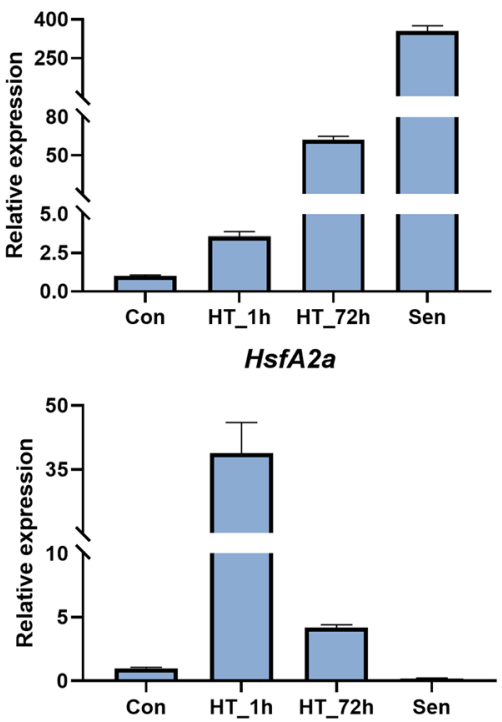

HsfA2a

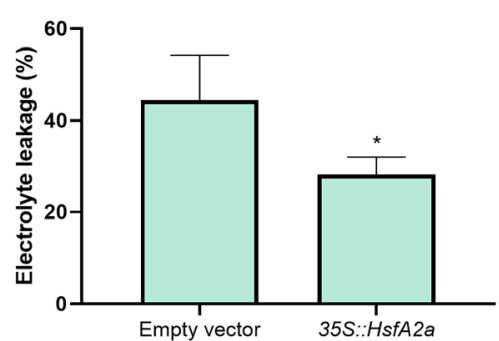

Fig. 9 Quantitative real time PCR (qRT-PCR) validation and function analysis of differentially expressed transcription factors. a Relative expression of NAM-B2 (i1_LQ_TF3rd_c8869/f1p0/1379), NAC029 (i1_HQ_TF3rd_C26766/f2p2/1431), NAM-B1 (iI_HQ_TF3rd_C44692/f2p0/1636), HsfA2d-1 (i1_LQ_TF3rd_c31016/f1p0/1406), HsfA2d-2 (i1_LQ_TF3rd_c80591/f1p0/1429), and HsfA2a (i3_HQ_TF3rd_c12860/f2p0/3466). b Membrane damages in agro-infiltrated Nicotiana. benthamiana plants with NAC029 (i1_HQ_TF3rd_c26766/f2p2/1431), NAM-B1 (i1_HQ_TF3rd_c44692/f2p0/1636), and HsfA2a (i3_HQ_TF3rd_c12860/f2p0/3466) after 1 day at $45^{\circ} \mathrm{C}$ by electrolyte leakage assay compared with agro-infiltrated plants with empty vector. Asterisks indicated significant difference with wild type at high temperature as estimated using Student's t-test (* $\left.P<0.05,{ }^{*} P<0.01\right)$. Error bars represent the standard error of the mean $(n=3)$ 
by RNA-seq, indicating that RNA-seq results were reliable.

\section{Transient expression of two NACs and one Hsfs in N. benthamiana leaves}

The function of three TFs, including NAC029, NAM-B1 and HsfA2a, were characterized in transient expression experiments conducted in $N$. benthamiana. The left half of the leaf was infiltrated with an empty vector, and the right half of the same leaf was infiltrated with a binary construct containing $35 \mathrm{~S}$ promoter and the coding sequence of each TF. After $24 \mathrm{~h}$ of heat treatment with $45^{\circ} \mathrm{C}$, cell membrane injury of each sample was measured by electrolyte leakage. As the results show (Fig. 9b), plants agro-infiltrated with the 35S::NAC029 or $35 S:: N A M-B 1$ constructs both exhibited significantly higher levels of cell membrane injury compared with controls, whereas plants agro-infiltrated with the $35 \mathrm{~S}:$ : $H s f A 2 a$ construct exhibited significantly lower levels of cell membrane injury compared with controls.

\section{Discussion}

HS is the key determinant of growth and development for the cool-season grass tall fescue [2, 3]. High temperatures can result in significant changes in the transcriptome, proteome, and metabolome. A comprehensive understanding of genome-wide HSR genes is the first and essential step to investigate the transcriptional regulatory network of HS response in tall fescue. In this study, SMRT sequencing combined with Illumina sequencing were firstly used in tall fescue. The SMRT sequencing showed a great improvement in mean length of unigenes and the ratio of full-length transcripts compared with the secondgeneration RNA sequencing [13]. A total of 62,443 unigenes with an average length of $2595 \mathrm{bp}$ in tall fescue were obtained here (Fig. 2). The high ratio of full-length transcripts contributes to further cloning of heat-responsive genes, as well as functional analysis.

\section{Common and distinct heat-responsive genes in short- and long-term heat stress}

In this study, tall fescue plants were treated by short- (1 h) and long-term (72 h) heat stress, respectively. Compared with previous studies in tall fescue $(2 \mathrm{~h}$ and $12 \mathrm{~h}$ ) and wheat $(1 \mathrm{~h}$ and $24 \mathrm{~h})[15,31]$, a relative small part of genes was co-regulated by short and prolonged heat treatments here. It could be the result of a long interval of two heat treatments. These commonly up-regulated DEGs mainly included Hsps, GSTs, and photosynthesisrelated genes. Hsps play a crucial role in protecting plants against HS by re-establishing normal protein conformation and thus cellular homeostasis [5]. Although the induction of Hsps during heat stress has been extensively reported [7, 32, 33], Hsps were just a fraction of heat-induced transcripts in previous studies [13, 34, 35]. There were only $7.75 \%$ (117/1509) of DEGs annotated as Hsps in wheat after $1 \mathrm{~h}$ of HS [31, 34]. Inconsistently, the Hsps made the largest proportion, almost half of the commonly up-regulated DEGs in tall fescue after heat treatments. In addition, the expression of these Hsps were altered more dramatically in HT_1h than in HT 72h, which is consistent with the previous research [31]. The result indicates that the response of Hsps to heat stress is extensive and quite fast. Heat stress produces ROS which is toxic to plant cell and affects the repair system of photosystem II [36, 37]. Several studies demonstrated that ROS-scavenging mechanisms have an important role in protecting plants against hightemperature stress [38]. In this study, ROS scavengers (GSTs) and genes involved in photosynthesis were both co-induced by HT_1h and HT_72h, implying that tall fescue plants try to scavenge ROS and maintain the steady photosynthesis to minimize the damages caused by high temperature.

Heat responses of tall fescue were distinct in terms of heat treatment time. Here, we found that the short-term heat response in tall fescue is a much quicker and a more dramatic process than ever reported. In the previous study, the number of DEGs under $2 \mathrm{~h}$ of short-term heat treatment (4311) was only half of that under $12 \mathrm{~h}$ of long-term heat treatment (8395), and most of the DEGs were downregulated [15]. However, in our study, much more DEGs were specifically regulated by HT_1h, and $76.48 \%$ of which were up-regulated more than 32 fold. The much quicker response of short-term HS was further highlighted by a study that showed that 42.83 and $74.50 \%$ DEGs in leaves and grain of wheat were detected within the first $30 \mathrm{~min}$ of heat treatment [39]. After $1 \mathrm{~h}$ of heat stress, half of the DEGs were Hsps; however, almost no Hsps were upregulated specifically by $\mathrm{HT}_{-} 72 \mathrm{~h}$, further indicating that the response of Hsps to heat stress is fast, short and drastic in tall fescue. FKBPs, another chaperone family with the classical role in protein folding [40], were also highly induced by HT_1h. Inconsistency with that FaFKBP65 was induced from 0 to 13.33 by HT_1h in this study, the expression of OsFKBP65 was detected at the RNA level only after heat stress [41]. In Arabidopsis, AtFKBP62 modulates thermotolerance by interacting with Hsp90.1 and affecting the accumulation of HsfA2-regulated sHsps [30]. Here, six FaFKBP62s were significantly induced by HT_1h. The results suggest that FKBPS are activated to resist short-term high temperature in tall fescue. Calmodulin has been reported to be involved in FKBP functioning, and the role of calcium signaling in HSR has been demonstrated in wheat and arabidopsis [7, 42, 43]. Consistently, PCM5 which encodes calmodulin was up-regulated by HT_1h. Besides PCM5, other calcium signaling genes were also identified after $1 \mathrm{~h}$ of heat treatment, suggesting a possible role of 
$\mathrm{Ca}^{2+}$ mediated signaling in short-term HSR. The roles of plant hormones, such as ABA, SA, and ethylene in thermotolerance have been well reported $[9,44]$. Here, genes involved in ABA, brassinosteroids, JA, and cytokinin were induced by short-term HS, although the roles these upregulated genes in heat stress have not been demonstrated. Additional experiments are needed to determine their roles and downstream pathways in thermotolerance.

The process of long-term HSR was quite different from that of short-term HSR. Compared with shortterm HSR, more genes were suppressed. After long-term heat stress, ribosomal proteins, $C Y P s$, and potassium transporters were significantly induced, while phytohormone signaling genes, calcium signaling genes, SWEETs, and 14-3-3s were suppressed. Ribosomal proteins accounted for the largest proportion of DEGs that were up-regulated specifically by HT_72h. Consistently, a large number of ribosomal protein genes were upregulated after HS, especially the long-term HS in wheat [31]. Given that ribosomal proteins are essential for protein synthesis, the induction of ribosomal proteins may indicate their role in preventing interference of protein synthesis by long-term heat stress. Potassium ion $\left(\mathrm{K}^{+}\right)$is an essential nutrient for life, and $\mathrm{K}^{+}$transport across membranes is mediated by the KT/HAK/KUP family [45]. KT/HAK/KUP transporters have been documented to participate in stress responses, including salt and drought $[46,47]$. However, the role of $K T / H A K / K U P$ transporters in heat stress has not been reported. Here, the upregulation of HAKs supplies a clue for further investigation of HAK-mediated heat response. They may rescue cellular osmotic imbalance caused by high temperature through $\mathrm{K}^{+}$uptake and efflux. In contrast to HT_1h, genes involved in plant hormone signal transduction, including auxin, cytokinin, ABA, ethylene, and JA were mainly suppressed by HT_72h. JA and ethylene were reported to have opposite roles in heat stress tolerance in Arabidopsis. The coil mutant is more susceptible to heat stress, whereas the ein 2 mutant shows increased thermotolerance $[48,49]$. However, the expressions of FaCOI1 and FaEIN2 were both reduced after HT_72h, indicating that the roles of COI1 and EIN2 in heat stress may be species-specific. 14-3-3 proteins function as regulators in various abiotic and biotic stresses [50]. In Drosophila cells, a 14-3-3 protein was observed to dissolve and re-naturalize thermal-aggregated proteins [51]. However, the function of 14-3-3 protein in HS has not been reported in plants. Here, we found that long-term HS was associated with 14-3-3 proteins in tall fescue. Several GF14s were suppressed specifically by HT_72h. In contrast, most of the 14-3-3 proteins showed a transient elevation of expression after $15 \mathrm{~min}$ of heat treatment in Arabidopsis [52].

\section{The synergetic and antagonistic relationships between} heat stress and senescence

The relationship between senescence and short- or longterm heat stress was also investigated in this study. There may be an antagonistic relationship between short-term HS and leaf senescence; however, short-term heat stress could accelerate leaf senescence. SAG12, a reference gene for leaf senescence, increases remarkably with age [53]. It was decreased by HT_1h but increased by HT_72h, indicating that leaf senescence was somewhat reduced after short-term HS, but induced after long-term HS. In previous studies, heat-induced leaf senescence was negatively associated with cytokinin synthesis, and positively associated with ethylene and ABA [18, 19]. Consistently, ethylene synthesis gene $A C O$ and cytokinin degradation gene $C K X 4$ were induced by both HT_72h and Sen. In addition, synthesis genes of another senescence-promoting phytohormone JA were also induced by HT_72h and Sen. Our results indicated that long-term heat stress could promote senescence by regulating genes involved in phytohormone synthesis and degradation. During leaf senescence, chlorophyll is degraded to colorless products via SGR and chlorophyll catabolic enzymes (CCEs), such as NYC1, NOL, PPH, PAO, and RCCR [54-56]. SGR-CCE-LHCII complexes are formed to trigger LHCPII destabilization for the degradation of Chls and Chl-free LHCPII subunits in senescing leaves [57]. Here, SGR and three CCEs were induced but LHCbs were suppressed by both HT_72h and Sen, indicating that long-term heat stress accelerates senescence through SGR-CCE-LHCII complexes.

\section{The responses of FaHsfs and FaNACs to heat stress and leaf senescence}

TFs play pivotal roles in response to diverse abiotic and biotic stresses. Here, members of heat-responsive TF families were identified. Hsfs are the master regulators of the HSR in all eukaryotic organisms [58]. Here, expression levels of $H s f A 2 s$ were the most strongly induced by HS, which was consistent with previous studies [12, 59]. Several studies have reported function analysis of HsfA2, but it is still under controversy [10, 60, 61]. In this study, N. benthamiana transiently overexpressing FaHs$f A 2 a$ displayed increased tolerance to HS. The further in vivo functional analysis of it will be carried out in tall fescue. Class B Hsfs were always supposed to have repressor functions during HS because of -LFGV-motifs. A small part of $H s f B 2 s$ was induced by short- or long-term $\mathrm{HS}$, and their functions need to be investigated further [61]. Few class C Hsfs have been identified in Arabidopsis or tomato, the function of which is still unknown. Consistently, only one $H s f C$ was induced by HS in tall fescue. NAC, a specific transcription factor family for plants, plays diverse roles in plant development and abiotic stresses 
[62]. Several NACs have been identified as positive regulators for senescence, interestingly, some of which are involved in plant stress tolerance as well $[63,64]$. Here, many of NACs were significantly induced by natural senescence and long-term HS, indicating their potentially important roles in heat-induced senescence. NAC029, a key regulator for natural senescence, has also been identified to be involved in salt, drought, and cold stresses [63, 6567]. However, the role of NAC029 in HS is still unknown. One tall fescue gene i1_HQ_TF3rd_c26766/f2p2/1431 which was annotated as NACO29 was found to be largely induced by both Sen and HT_72h. N. benthamiana transiently overexpressing FaNAC029 displayed increased membrane injury, suggesting that FaNACO29 may play an important role in senescence accelerated by long-term HS in tall fescue. Meanwhile, functional analysis of another NAC gene i1_HQ_TF3rd_c44692/f2p0/1636 which was annotated as $N A M-B 1$ was performed in $N$. benthamiana as well. It was reported that $N A M-B 1$ could accelerate senescence in wheat [68], and post-anthesis heat and a $N A M-B 1$ introgression have similar (both could accelerate peduncle senescence) but non-additive effects in bread wheat [69]. However, in this study, the leaf infected with $35 S:: N A M-B 1$ showed more significant membrane injury after HS compared with that infected with an empty vector, indicating that FaNAM-B1 may play an important role in senescence accelerated by long-term HS in tall fescue. Further in vivo functional analysis of these two NAC genes will be carried out in tall fescue.

The involvement of AS in heat stress and leaf senescence AS is involved in many plant processes, especially responses to environmental stresses [70]. In this study, we only detected 298 AS events responsive to HS, the number of which was much less than that in other studies [71]. This may be due to the lack of a reference genome sequence. However, the distribution of AS events in tall fescue is consistent with other plants in which RI is the major AS event [29]. It is well documented that Hsfs and $H$ sps are easily alternatively spliced [26, 72]. Differentially expressed $H s p s$ and $H s f s$ isoforms generated by AS were widely observed after heat treatment in tall fescue. In addition, many other isoforms, such as SGR, PAO, and PED1 produced by AS were also differentially expressed during heat stress in tall fescue. Although no study investigating the role of AS in senescence was reported in plants, NAC genes, ELF3s and AAO3 generated by AS were observed in senescent leaves in tall fescue. The function of AS for these genes during HS and senescence needs further investigation.

\section{Conclusions}

The short- and long-term HSR and the relationship between HS and senescence were investigated in this study
(Fig. 10). Our results showed that the short-term HS can activate a large number of $H s p s$, FKBPs, calcium signaling genes, phytohormone signaling genes, and $H s f s$ to improve thermotolerance. However, long-term HS may lead to leaf senescence via activating chlorophyll catabolic genes, phytohormone synthesis/degradation genes, stress-related genes and, NAC TFs, and suppressing photosynthesis-related genes, FKBPS and CATs. During heat stress and senescence, a large number of HS- and senescence-associated DEIs were generated by AS. Further studies involving in-depth characterizations of heatresistance breeding candidate genes in tall fescue are required to reveal the underlying mechanism of heat adaptation and heat-induced senescence.

\section{Methods}

Plants materials and treatments

Seeds of commercial-type tall fescue 'Houndog 5' with relatively high thermotolerance (Additional file 13) [73] provided by Baisite Company (Beijing) were sown in plastic pots $(10 \mathrm{~cm}$ diameter, $10 \mathrm{~cm}$ height $)$ with a mixture of sand and peat soil $(1 / 2, v / v)$. Pots were then maintained in the greenhouse at a temperature of $22^{\circ} \mathrm{C} /$ $18^{\circ} \mathrm{C}$ (day/night), a photoperiod of $14 \mathrm{~h}$ light $/ 10 \mathrm{~h}$ dark, and relative humidity of $70-80 \%$. After 2 months of establishment, all plants were transferred into controlledenvironment growth chambers (HP300GS-C, Ruihua Instrument, Wuhan, China), with a $16 \mathrm{~h}$ photoperiod, photosynthetically active radiation at $450 \mu \mathrm{mol} \mathrm{m}^{-2} \mathrm{~s}^{-1}$ at the canopy level, a day/night temperature of $22 / 18^{\circ} \mathrm{C}$, and $70 \%$ relative humidity. The plants acclimated in this condition for 1 week before they were exposed to heat treatments. After one weak, green leaves of tall fescue were collected as control (named as Con). Then the remaining plants were divided into two groups. Group I of tall fescues were kept at $22 / 18^{\circ} \mathrm{C}$ (day/night) in the controlled-environment growth chambers until the leaves of tall fescues appeared senescent, and the senescent leaves were collected (named as Sen). Group II was treated with $38^{\circ} \mathrm{C}$ day $/ 33^{\circ} \mathrm{C}$ night in the controlled-environment growth chambers. In addition to temperature, other growth environment was as same as control. Leaves were collected after $1 \mathrm{~h}$ (named as HT_1h) and $72 \mathrm{~h}$ (named as HT_72h) of heat treatments, respectively. All collected samples were immediately frozen in liquid nitrogen and stored at $-80^{\circ} \mathrm{C}$ until RNA isolation. All the treatments were repeated for three replications.

\section{RNA preparation and Illumina RNA sequencing}

Total RNA was extracted using the Trizol kit (Invitrogen, Carlsbad, CA) from the collected samples (Con, Sen, HT_1h, and HT_72h). Quantity and quality of the total RNA were detected by NanoDrop 2000 (Thermo, Waltham, USA) and a $1.2 \%$ agarose gel. A total of $1.5 \mu \mathrm{g}$ 


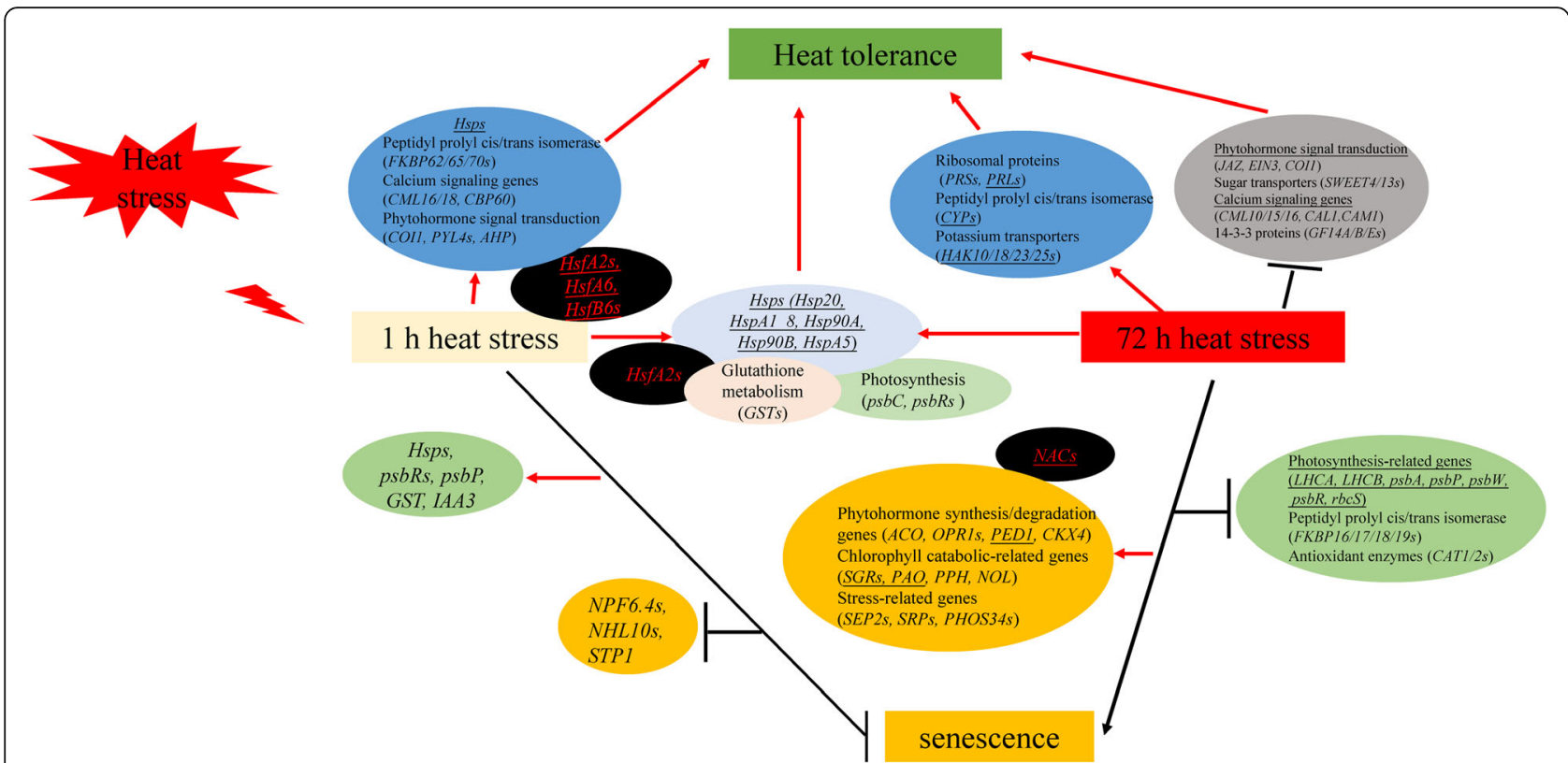

Fig. 10 A hypothetical model of heat stress response and the crosstalk between heat stress and leaf senescence in tall fescue. Underlines represent that the differentially expressed isoforms were produced by AS

RNA per sample was used for Illumina RNA sequencing. Sequencing libraries were constructed using NEBNext ${ }^{\circ}$ Ultra $^{\text {tw }}$ RNA Library Prep Kit for Illumina ${ }^{\circ}$ (NEB, Ipswich, USA) according to the manufacturer's recommendations. Library quality was evaluated on the Agilent Bioanalyzer 2100 system.

\section{PacBio SMRT sequencing of pooled RNA samples}

For PacBio SMRT sequencing, a pooled RNA sample was mixed from 12 RNA samples used for Illumina RNA-seq with equal amounts. The Isoform Sequencing (Iso-Seq) library was generated following the Iso-Seq protocol using the Clontech SMARTer PCR cDNA Synthesis Kit (Takara, Dalian, China) and the BluePippin Size Selection System protocol which is described by $\mathrm{Pa}-$ cific Biosciences (PN 100-092-800-03).

\section{PacBio data processing}

After PacBio sequencing, raw data were processed using the SMRTlink 6.0 software. CCSs were generated from subreads with passes 2 , accuracy $>0.8$, and max_length of 15,000 . According to whether there were $5^{\prime}-$ and $3{ }^{\prime}$ cDNA primers, and whether there was a poly-A tail signal preceding the 3 '-primer, a CCS or subread sequence was divided into full length, non-full length, and chimeric reads. Full-length and non-full length fasta data were subsequently inputted into the clustering step, which performs isoform-level clustering by ICE, followed by Arrow polishing finally. To improve the accuracy of consensus, additional nucleotide errors of consensus reads were further corrected using the Illumina sequencing data by
LoRDEC [65]. Final transcripts were obtained through removing redundancy in corrected consensus by CD-HIT.

\section{Full-length unique transcript model reconstruction}

The non-redundant transcripts were processed with the Coding GENome reconstruction tool (Cogent v3.1, https:// github.com/Magdoll/Cogent) with the parameters: --dun_ use_partial. In general, Cogent firstly split the input fasta file into chunks of chunk_size and computed the k-mer profile. Each transcript family was further reconstructed into one or several unique transcript models (referred to as UniTransModels) using a De Bruijn graph method.

\section{Alternative splicing analysis}

Error-corrected non-redundant transcripts (transcripts before Cogent reconstruction) were mapped to UniTransModels using gmap-2017-06-20. Splicing junctions for transcripts mapped to the same UniTransModels were examined, and transcripts with the same splicing junctions were collapsed. Collapsed transcripts with different splicing junctions were identified as transcription isoforms of UniTransModels. AS events were detected with SUPPA (https://github.com/comprna/SUPPA) using default settings. Quantification of the differential expression of AS events were carried out by rMATS [74]. The significant differential expression of AS events were filtered with $|\mathrm{dpsi}|>$ 0.1 and $p$-value $<0.05$;

Differential expression analysis based on Illumina data Gene expression levels of each sample were calculated by RSEM [75]. DESeq R package (1.10.1) was used for 
differential expression analysis of two groups. DESeq provided statistical routines for determining differential expression in digital gene expression data using a model based on the negative binomial distribution. The resulting $P$ values were adjusted using the Benjamini and Hochberg's approach for controlling the false discovery rate. A gene was regarded as a DEG if it showed a twofold difference, $\mid \log _{2}$ Ratio (Treatment/Control) $\mid \geq 1$, the adjusted $p$-value $\leq 0.05$, and FPKM $>10$ in either sample of each comparison.

\section{Quantitative real-time PCR analysis of DEGs}

The expression levels of DEGs were performed by qRTPCR following the previously described method [76]. In brief, cDNA synthesis was performed using a PrimeScript RT reagent kit (Takara, Dalian, China). The qRT-PCR was performed using $20 \mu \mathrm{l}$ volumes of SYBR (Takara, Dalian, China) and the ABI STEPONE Real-Time PCR system. FaACTIN was selected as a reference gene for normalization. All gene-specific primers used for qRT-PCR analysis are listed in Additional file 14. Three independent biological replicates were used for qRT-PCR analysis.

\section{Transient expression in $N$. benthamiana and electrolyte leakage assay}

For the transient overexpression experiments, plasmids were transferred to Agrobacterium tumefaciens strain AGL1. Agroinfiltration was performed as described previously $[77,78]$. Briefly, overnight-grown bacterial cultures were re-suspended in agro-infiltration medium (10 $\mathrm{mM}$ MES, pH 5.6, $10 \mathrm{mM} \mathrm{MgCl}_{2}$, and $200 \mu \mathrm{M}$ acetosyringone) to OD600 of 1.0. Needle-free syringes were used to infiltrate leaves of $N$. benthamiana plants under growing conditions with $24{ }^{\circ} \mathrm{C}$ day $/ 20^{\circ} \mathrm{C}$ night in a $16 \mathrm{~h}$ light $/ 8 \mathrm{~h}$-dark cycle. After infiltration, the plants were kept at $22^{\circ} \mathrm{C}$ overnight. The following morning, the agro-infiltrated plants were moved to $45^{\circ} \mathrm{C}$. After $24 \mathrm{~h}$ (in a $12 \mathrm{~h}$-light $/ 12 \mathrm{~h}$-dark cycle) of high-temperature treatment, leaves were harvested for EL assays. Three replicate samples of five $1 \mathrm{~cm}^{2}$ leaf discs were punched from each leaf sample and then were washed twice with deionized water. The discs were placed in a $50 \mathrm{~mL}$ tube. A total of $25 \mathrm{~mL}$ of deionized water was added into each tube, and then samples were kept in a $25^{\circ} \mathrm{C}$ shaker with $150 \mathrm{rpm}$. After $4 \mathrm{~h}$, the initial conductivity was measured using a conductivity meter. Then samples were autoclaved, and total conductivity was determined after cooling to room temperature. The extent of cell membrane injury was calculated as follows: Initial conductivityx 100/total conductivity.

\section{Measurements of Fv/Fm}

Before measuring $\mathrm{Fv} / \mathrm{Fm}$, leaves were kept in the dark for $30 \mathrm{~min}$ to close all PSII reaction centers. Then, $\mathrm{Fv} /$
Fm was measured by a pulse-amplitude modulation (PAM) portable chlorophyll fluorometer (PAM-2500, WALZ, Effeltrich, Germany) according to the manufacturer's protocol.

\section{Supplementary information}

Supplementary information accompanies this paper at https://doi.org/10. 1186/s12870-020-02572-4.

Additional file 1. Numbers and distribution rate of unigenes in seven databases.

Additional file 2. Functional annotation of the full-length transcriptome in tall fescue.

Additional file 3. The differential expression of DEGs in comparison V4. Additional file 4. KEGG analysis of DEGs and DEls specifically induced by HT_1h.

Additional file 5. The differential expression of DEGs in comparison $\mathrm{V} 1$. Additional file 6. KEGG analysis of DEGs specifically regulated by HT_72h.

Additional file 7. The differential expression of DEGs in comparison V2. Additional file 8. The differential expression of DEGs in comparison V5. Additional file 9. KEGG analysis of DEGs down-regulated by HT_72h and Sen.

Additional file 10. KEGG analysis of DEGs specifically regulated by natural senescence.

Additional file $\mathbf{1 1}$ The differential expression of Hsfs in each comparison.

Additional file 12. The differential expression of NAC transcription factors in each comparison.

Additional file 13. The phenotypic differences between 'Houndog 5' (heat-tolerant) and 'PI535582' (heat-sensitive) after heat treatments.

Additional file 14. All primers used in this study.

\section{Abbreviations}

HS: Heat stress; DEG: Differentially expressed gene; Hsp: Heat shock proteins; Hsf: Heat shock factors; ROS: Reactive oxygen species; APX: Ascorbate peroxidase; TF: Transcription factors; SMRT: Single-molecular real-time; AS: Alternative splicing; PacBio: Pacific Biosciences; CCS: Circular consensus sequences; FLNC: Full-length non-chimeric read; ICE: Iterative Clustering for Error Correction; NFL: Non-full-length; Nr: Non-redundant protein sequence database; Nt: Nucleotide sequence database; KOG/COG: Clusters of orthologous groups of proteins; KEGG: Kyoto encyclopedia of genes and genomes; GO: Gene ontology; Con: Control; HT_1h: $1 \mathrm{~h}$ of $38^{\circ} \mathrm{C}$ heat treatment; HT_ $72 \mathrm{~h}$ : $72 \mathrm{~h}$ of $38^{\circ} \mathrm{C}$ heat treatment; Sen: Senescent leaves; GST: Glutathione S-transferase; ABA: Abscisic acid; JA: Jasmonic acid; SE: Skipping exon; MX: Mutually exclusive exon; A5: Alternative 5' splice-site; A3: Alternative 3' splice-site; Rl: Retained intron; AF: Alternative first exon; AL: Alternative last exon; DEASE: Differentially expressed AS events; IsoSeq: Isoform Sequencing; FKBP: FK506-binding Protein; CATs: Catalases; SGR: Staygreen; EL: Electrolyte Leakage; DEI: Differentially Expressed Isoforms; PRS: Small Subunit Ribosomal Protein; PRL: Large Subunit Ribosomal Proteins; PAO: Pheophorbide A Oxygenase; PPH: Pheophytinase; NOL: Chlorophyll b Reductase; ACO: 1-Aminocyclopropane-1-Carboxylate Oxidase; OPR1: 12-

Oxophytodienoate Reductase 1; PED1: Peroxisome Defective 1;

CKX4: Cytokinin Oxidase/Dehydrogenase 4; SEP2: Stress Enhanced Protein 2; SRP: Stress-Related Proteins; ELF3: Early Flowering 3; AAO3: Abscisic Aldehyde Oxidase 3; HSR: HS Response; CCE: Chlorophyll Catabolic Enzyme

Acknowledgements

Not applicable.

Authors' contributions

YGQ and LWC wrote this manuscript and conducted experiments. YGQ,

LWC, and QZ analyzed sequencing data, MA analyzed sequencing data and 
revised the manuscript, and KC and LC designed the experiment, provided guidance and revised the manuscript. All authors read and approved the manuscript.

\section{Funding}

This work was financially supported by the National Natural Science Foundation of China (41503067 and 31672482), the National Science Foundation for Young Scientists of China (41603121), the Fundamental Research Funds for the Central University (CZZ19006), the Major Science and Technology Innovation Project of Shandong Province (2019JZZY010726), Poverty Alleviation through Agricultural Projects from the Agricultural Office of Chinese Academy of Sciences, and the Strategic Priority Research Program of the Chinese Academy of Sciences. These funding bodies did not play any roles in the design of the study and collection, analysis, and interpretation of data and in writing the manuscript.

\section{Availability of data and materials}

The datasets generated and analyzed during the current study are available in the NCBI Sequence Read Archive repository under Bioproject PRJNA647166 (https://www.ncbi.nlm.nih.gov/Traces/study/?acc=PRJNA64 7166).

\section{Ethics approval and consent to participate} Not applicable.

\section{Consent for publication}

Not applicable.

\section{Competing interests}

The authors declare that they have no competing interests.

\section{Author details}

${ }^{1}$ Shenzhen Key Laboratory of Environmental Chemistry and Ecological Remediation, College of Chemistry and Environmental Engineering, Shenzhen University, Shenzhen, People's Republic of China. ${ }^{2}$ CAS Key Laboratory of Plant Germplasm Enhancement and Specialty Agriculture, Wuhan Botanical Garden, The Innovative Academy of Seed Design, Chinese Academy of Sciences, Wuhan, People's Republic of China. ${ }^{3}$ College of Resources and Environmental Science, Key Laboratory of Catalysis and Energy Materials Chemistry of Ministry of Education \& Hubei Key Laboratory of Catalysis and Materials Science, South-Central University for Nationalities, Wuhan, People's Republic of China. ${ }^{4}$ Center of Economic Botany, Core Botanical Gardens, Chinese Academy of Sciences, Wuhan, People's Republic of China.

\section{Received: 8 October 2019 Accepted: 23 July 2020}

Published online: 03 August 2020

\section{References}

1. Wang Z, Hopkins A, Lawrence R, Bell J, Scott M. Field evaluation and risk assessment of transgenic tall fescue (Festuca arundinacea) plants. In: Molecular breeding of forage and turf: Dordrecht: Springer; 2004:367-79.

2. Jiang $Y$, Huang B. Physiological responses to heat stress alone or in combination with drought: a comparison between tall fescue and perennial ryegrass. HortScience. 2001;36(4):682-6.

3. Wang J, Cui L, Wang Y, Li J. Growth, lipid peroxidation and photosynthesis in two tall fescue cultivars differing in heat tolerance. Biol Plant. 2009;53(2): 237-42

4. Moreno A, Orellana A. The physiological role of the unfolded protein response in plants. Biol Res. 2011;44(1):75-80.

5. Wang W, Vinocur B, Shoseyov O, Altman A. Role of plant heat-shock proteins and molecular chaperones in the abiotic stress response. Trends Plant Sci. 2004;9(5):244-52

6. Miller G, Mittler R. Could heat shock transcription factors function as hydrogen peroxide sensors in plants? Ann Bot. 2006;98(2):279-88.

7. Kotak S, Larkindale J, Lee U, von Koskull-Döring P, Vierling E, Scharf K. Complexity of the heat stress response in plants. Curr Opin Plant Biol. 2007; 10(3):310-6.

8. Ohama N, Sato H, Shinozaki K, Yamaguchi-Shinozaki K. Transcriptional regulatory network of plant heat stress response. Trends Plant Sci. 2017; 22(1):53-65.
9. Larkindale J, Huang B. Thermotolerance and antioxidant systems in Agrostis stolonifera: involvement of salicylic acid, abscisic acid, calcium, hydrogen peroxide, and ethylene. J Plant Physiol. 2004;161(4):405-13.

10. Schramm F, Ganguli A, Kiehlmann E, Englich G, Walch D, von Koskull-Döring $P$. The heat stress transcription factor HsfA2 serves as a regulatory amplifier of a subset of genes in the heat stress response in Arabidopsis. Plant Mol Biol. 2006;60(5):759-72.

11. Larkindale J, Hall J, Knight M, Vierling E. Heat stress phenotypes of Arabidopsis mutants implicate multiple signaling pathways in the acquisition of thermotolerance. Plant Physiol. 2005;138(2):882-97.

12. Wang $Y$, Dai $Y$, Tao X, Wang J, Cheng H, Yang H, Ma X. Heat shock factor genes of tall fescue and perennial ryegrass in response to temperature stress by RNA-Seq analysis. Front Plant Sci. 2016;6:1226.

13. Hu T, Sun $X$, Zhang $X$, Nevo E, Fu J. An RNA sequencing transcriptome analysis of the high-temperature stressed tall fescue reveals novel insights into plant thermotolerance. BMC Genomics. 2014;15(1):1147.

14. Zhang Y, Mian MR, Chekhovskiy K, So S, Kupfer D, Lai H, Roe BA. Differential gene expression in Festuca under heat stress conditions. J Exp Bot. 2005; 56(413):897-907.

15. Alam M, Zhang L, Yang L, Islam M, Liu Y, Luo H, Yang P, Wang Q, Chan Z. Transcriptomic profiling of tall fescue in response to heat stress and improved thermotolerance by melatonin and 24-epibrassinolide. BMC Genomics. 2018;19(1):224.

16. Wang $X$, Huang W, Liu J, Yang Z, Huang B. Molecular regulation and physiological functions of a novel FaHsfA2c cloned from tall fescue conferring plant tolerance to heat stress. Plant Biotechnol J. 2017;15(2):237-48.

17. Kim K, Alam I, Kim Y, Sharmin S, Lee K, Lee S, Lee B. Overexpression of a chloroplast-localized small heat shock protein OsHSP26 confers enhanced tolerance against oxidative and heat stresses in tall fescue. Biotechnol Lett. 2012:34(2):371-7.

18. $X u$ Y, Huang B. Heat-induced leaf senescence and hormonal changes for thermal bentgrass and turf-type bentgrass species differing in heat tolerance. J Am Soc Hortic Sci. 2007;132(2):185-92.

19. Xing J, Xu Y, Tian J, Gianfagna T, Huang B. Suppression of shade-or heatinduced leaf senescence in creeping bentgrass through transformation with the ipt gene for cytokinin synthesis. J Am Soc Hortic Sci. 2009;134(6):602-9.

20. Jespersen D, Yu J, Huang B. Metabolite responses to exogenous application of nitrogen, cytokinin, and ethylene inhibitors in relation to heat-induced senescence in creeping bentgrass. PLoS One. 2015;10(3):e0123744.

21. Jespersen D, Huang B. Proteins associated with heat-induced leaf senescence in creeping bentgrass as affected by foliar application of nitrogen, cytokinins, and an ethylene inhibitor. Proteomics. 2015;15(4):798-812.

22. Meshorer $\mathrm{E}$, Soreq $\mathrm{H}$. Pre-mRNA splicing modulations in senescence. Aging Cell. 2002;1(1):10-6.

23. Blanco F, Bernabeu C. Alternative splicing factor or splicing factor-2 plays a key role in intron retention of the endoglin gene during endothelial senescence. Aging Cell. 2011;10(5):896-907.

24. Guerra D, Crosatti C, Khoshro H, Mastrangelo A, Mica E, Mazzucotelli E. Posttranscriptional and post-translational regulations of drought and heat response in plants: a spider's web of mechanisms. Front Plant Sci. 2015;6:57.

25. Fujikake N, Nagai Y, Popiel $H$, Kano H, Yamaguchi M, Toda T. Alternative splicing regulates the transcriptional activity of Drosophila heat shock transcription factor in response to heat/cold stress. FEBS Lett. 2005:579(17):3842-8.

26. Liu J, Sun N, Liu M, Liu J, Du B, Wang X, Qi X. An autoregulatory loop controlling Arabidopsis HsfA2 expression: role of heat shock-induced alternative splicing. Plant Physiol. 2013;162(1):512-21.

27. Mian M, Hopkins A, Zwonitzer J. Determination of genetic diversity in tall fescue with AFLP markers. Crop Sci. 2002;42(3):944-50.

28. Sharon D, Tilgner $H$, Grubert F, Snyder M. A single-molecule long-read survey of the human transcriptome. Nat Biotechnol. 2013;31(11):1009.

29. Abdel-Ghany S, Hamilton M, Jacobi J, Ngam P, Devitt N, Schilkey F, Ben-Hur A, Reddy A. A survey of the sorghum transcriptome using single-molecule long reads. Nat Commun. 2016;7:11706

30. Meiri D, Breiman A. Arabidopsis ROF1 (FKBP62) modulates thermotolerance by interacting with HSP90. 1 and affecting the accumulation of HSfA2regulated sHSPs. Plant J. 2009:59(3):387-99.

31. Qin D, Wu H, Peng H, Yao Y, Ni Z, Li Z, Zhou C, Sun Q. Heat stress-responsive transcriptome analysis in heat susceptible and tolerant wheat (Triticum aestivum L.) by using Wheat Genome Array. BMC Genomics. 2008;9(1):432.

32. Fragkostefanakis $S$, Roeth $S$, Schleiff $E$, Scharf $K$. Prospects of engineering thermotolerance in crops through modulation of heat stress transcription 
factor and heat shock protein networks. Plant Cell Environ. 2015;38(9):188195.

33. Guo R, Wang X, Han X, Li W, Liu T, Chen B, Chen X, Wang-Pruski G. Comparative transcriptome analyses revealed different heat stress responses in high-and low-GS Brassica alboglabra sprouts. BMC Genomics. 2019;20(1):269.

34. Mittler R, Finka A, Goloubinoff P. How do plants feel the heat? Trends Biochem Sci. 2012;37(3):118-25.

35. Yan J, Yu L, Xuan J, Lu Y, Lu S, Zhu W. De novo transcriptome sequencing and gene expression profiling of spinach (Spinacia oleracea L.) leaves under heat stress. Sci Rep. 2016;6:19473.

36. Gururani M, Venkatesh J, Tran L. Regulation of photosynthesis during abiotic stress-induced photoinhibition. Mol Plant. 2015;8(9):1304-20.

37. Allakhverdiev S, Kreslavski V, Klimov V, Los D, Carpentier R, Mohanty P. Heat stress: an overview of molecular responses in photosynthesis. Photosynth Res. 2008:98(1-3):541.

38. Suzuki N, Mittler R. Reactive oxygen species and temperature stresses: a delicate balance between signaling and destruction. Physiol Plant. 2006; 126(1):45-51.

39. Wang X, Chen S, Shi X, Liu D, Zhao P, Lu Y, Cheng Y, Liu Z, Nie X, Song W. Hybrid sequencing reveals insight into heat sensing and signaling of bread wheat. Plant J. 2019;98(6): 1015-32.

40. Geisler M, Bailly A. Tete-a-Tete: the function of FKBPs in plant development. Trends Plant Sci. 2007;12(10):465-73.

41. Magiri E, Farchi-Pisanty O, Avni A, Breiman A. The expression of the large rice FK506 binding proteins (FKBPs) demonstrate tissue specificity and heat stress responsiveness. Plant Sci. 2006;170(4):695-704.

42. Liu H, Sun D, Zhou R. Ca ${ }^{2+}$ and AtCaM3 are involved in the expression of heat shock protein gene in Arabidopsis. Plant Cell Environ. 2005;28(10):1276-84.

43. Liu H, Li B, Shang Z, Li X, Mu R, Sun D, Zhou R. Calmodulin is involved in heat shock signal transduction in wheat. Plant Physiol. 2003;132(3):1186-95.

44. Robertson A, Ishikawa M, Gusta L, MacKenzie S. Abscisic acid-induced heat tolerance in Bromus Inermis Leyss cell-suspension cultures (heat-stable, abscisic acid-responsive polypeptides in combination with sucrose confer enhanced thermostability). Plant Physiol. 1994;105(1):181-90.

45. Li W, Xu G, Alli A, Yu L. Plant HAK/KUP/KT K+ transporters: function and regulation. In: Seminars in cell \& developmental biology. Amsterdam: Elsevier Academic Press; 2018. p.133-41.

46. Chen G, Hu Q, Luo L, Yang T, Zhang S, Hu Y, Yu L, Xu G. Rice potassium transporter $\mathrm{O} S \mathrm{HAK} 1$ is essential for maintaining potassium-mediated growth and functions in salt tolerance over low and high potassium concentration ranges. Plant Cell Environ. 2015;38(12):2747-65.

47. Osakabe Y, Arinaga N, Umezawa T, Katsura S, Nagamachi K, Tanaka H, Ohiraki H, Yamada K, Seo S, Abo M. Osmotic stress responses and plant growth controlled by potassium transporters in Arabidopsis. Plant Cell. 2013; 25(2):609-24.

48. Clarke S, Cristescu S, Miersch O, Harren F, Wasternack C, Mur L. Jasmonates act with salicylic acid to confer basal thermotolerance in Arabidopsis thaliana. New Phytol. 2009;182(1):175-87.

49. Kazan K. Diverse roles of jasmonates and ethylene in abiotic stress tolerance. Trends Plant Sci. 2015;20(4):219-29.

50. Roberts M, Salinas J, Collinge D. 14-3-3 proteins and the response to abiotic and biotic stress. Plant Mol Biol. 2002;50(6):1031-9.

51. Yano M, Nakamuta S, Wu X, Okumura Y, Kido H. A novel function of 14-3-3 protein: $14-3-3 \zeta$ is a heat-shock-related molecular chaperone that dissolves thermal-aggregated proteins. Mol Biol Cell. 2006;17(11):4769-79.

52. Dobrá J, Černý M, Štorchová H, Dobrev P, Skalák J, Jedelský P, Lukšanová H, Gaudinová A, Pešek B, Malbeck J. The impact of heat stress targeting on the hormonal and transcriptomic response in Arabidopsis. Plant Sci. 2015;231:52-61.

53. Lohman K, Gan S, John M, Amasino R. Molecular analysis of natural leaf senescence in Arabidopsis thaliana. Physiol Plant. 1994;92(2):322-8.

54. Hörtensteiner S. Stay-green regulates chlorophyll and chlorophyll-binding protein degradation during senescence. Trends Plant Sci. 2009;14(3):155-62.

55. Pružinská A, Tanner G, Aubry S, Anders I, Moser S, Müller T, Ongania K, Kräutler B, Youn J, Liljegren S. Chlorophyll breakdown in senescent Arabidopsis leaves. Characterization of chlorophyll catabolites and of chlorophyll catabolic enzymes involved in the degreening reaction. Plant Physiol. 2005;139(1):52-63.

56. Kusaba M, Ito H, Morita R, lida S, Sato Y, Fujimoto M, Kawasaki S, Tanaka R, Hirochika H, Nishimura M. Rice NON-YELLOW COLORING1 is involved in light-harvesting complex II and grana degradation during leaf senescence. Plant Cell. 2007;19(4):1362-75.
57. Park S, Yu J, Park J, Li J, Yoo S, Lee N, Lee S, Jeong S, Seo H, Koh H. The senescence-induced staygreen protein regulates chlorophyll degradation. Plant Cell. 2007;19(5):1649-64.

58. Baniwal S, Bharti K, Chan K, Fauth M, Ganguli A, Kotak S, Mishra S, Nover L, Port M, Scharf K. Heat stress response in plants: a complex game with chaperones and more than twenty heat stress transcription factors. J Biosci. 2004;29(4):471-87.

59. Busch W, Wunderlich M, Schöffl F. Identification of novel heat shock factordependent genes and biochemical pathways in Arabidopsis thaliana. Plant J. 2005;41(1):1-14.

60. Li C, Chen Q, Gao X, Qi B, Chen N, Xu S, Chen J, Wang X. AtHsfA2 modulates expression of stress responsive genes and enhances tolerance to heat and oxidative stress in Arabidopsis. Sci China Ser C Life Sci. 2005;48(6):540.

61. Ogawa D, Yamaguchi K, Nishiuchi T. High-level overexpression of the Arabidopsis HsfA2 gene confers not only increased themotolerance but also salt/osmotic stress tolerance and enhanced callus growth. J Exp Bot. 2007; 58(12):3373-83.

62. Wu A, Allu A, Garapati P, Siddiqui H, Dortay H, Zanor M, Asensi-Fabado M, Munné-Bosch S, Antonio C, Tohge T. JUNGBRUNNEN1, a reactive oxygen species-responsive NAC transcription factor, regulates longevity in Arabidopsis. Plant Cell. 2012;24(2):482-506.

63. Seok H, Woo D, Nguyen L, Tran H, Tarte V, Mehdi S, Lee S, Moon Y. Arabidopsis AtNAP functions as a negative regulator via repression of AREB1 in salt stress response. Planta. 2017;245(2):329-41.

64. Balazadeh S, Siddiqui H, Allu A, Matallana-Ramirez L, Caldana C, Mehrnia M, Zanor M, Köhler B, Mueller-Roeber B. A gene regulatory network controlled by the NAC transcription factor ANAC092/AtNAC2/ORE1 during saltpromoted senescence. Plant J. 2010;62(2):250-64.

65. Salmela L, Rivals E. LoRDEC: accurate and efficient long read error correction. Bioinformatics. 2014:30(24):3506-14.

66. Xu Z, Wang C, Xue F, Zhang H, Ji W. Wheat NAC transcription factor TaNAC29 is involved in response to salt stress. Plant Physiol Biochem. 2015; 96:356-63.

67. Guo Y, Gan S. AtNAP, a NAC family transcription factor, has an important role in leaf senescence. Plant J. 2006;46(4):601-12.

68. Uauy C, Distelfeld A, Fahima T, Blechl A, Dubcovsky J. A NAC gene regulating senescence improves grain protein, zinc, and iron content in wheat. Science. 2006;314(5803):1298-301.

69. Maphosa L, Collins N, Taylor J, Mather D. Post-anthesis heat and a Gpc-B1 introgression have similar but non-additive effects in bread wheat. Funct Plant Biol. 2014;41(9):1002-8.

70. Staiger D, Brown J. Alternative splicing at the intersection of biological timing, development, and stress responses. Plant Cell. 2013;25(10):3640-56.

71. Zhang Y, Nyong A, Maraga T, Shi T, Yang P. The complexity of alternative splicing and landscape of tissue-specific expression in lotus (Nelumbo nucifera) unveiled by Illumina-and single-molecule real-time-based RNAsequencing. DNA Res. 2019;26(4):301-11.

72. Keller M, Hu Y, Mesihovic A, Fragkostefanakis S, Schleiff E, Simm S. Alternative splicing in tomato pollen in response to heat stress. DNA Res. 2017;24(2):205-17.

73. Gong X, Wang M, Yan N. A comparative study on turf quality of tall fescue in Huaibei area [J]. Pratacultural Science. 2013;30(4):553-6.

74. Shen S, Park J, Lu Z, Lin L, Henry M, Wu Y, Zhou Q, Xing Y. rMATS: robust and flexible detection of differential alternative splicing from replicate RNASeq data. Proc Natl Acad Sci. 2014;111(51):E5593-601.

75. Li B, Dewey C. RSEM: accurate transcript quantification from RNA-Seq data with or without a reference genome. BMC Bioinformatics. 2011;12(1):323.

76. Cao L, Yu N, Li J, Qi Z, Wang D, Chen L. Heritability and reversibility of DNA methylation induced by in vitro grafting between Brassica juncea and B oleracea. Sci Rep. 2016;6:27233.

77. Trapero-Mozos A, Morris W, Ducreux L, McLean K, Stephens J, Torrance L, Bryan G, Hancock R, Taylor M. Engineering heat tolerance in potato by temperature-dependent expression of a specific allele of HEAT-SHOCK COGNATE 70. Plant Biotechnol J. 2018;16(1):197-207.

78. Wydro M, Kozubek E, Lehmann P. Optimization of transient agrobacteriummediated gene expression system in leaves of Nicotiana benthamiana. Acta Biochim Polon. 2006;53(2):289.

\section{Publisher's Note}

Springer Nature remains neutral with regard to jurisdictional claims in published maps and institutional affiliations. 